The equivariant $K$-theory and cobordism rings of divisive weighted projective spaces

Harada, Megumi and Holm, Tara and Ray, Nigel and Williams, Gareth

2013

MIMS EPrint: 2015.62

Manchester Institute for Mathematical Sciences

School of Mathematics

The University of Manchester

\footnotetext{
Reports available from: http://eprints.maths.manchester.ac.uk/

And by contacting: The MIMS Secretary

School of Mathematics

The University of Manchester

Manchester, M13 9PL, UK
} 


\title{
THE EQUIVARIANT $K$-THEORY AND COBORDISM RINGS OF DIVISIVE WEIGHTED PROJECTIVE SPACES
}

\author{
MEGUMI HARADA, TARA S. HOLM, NIGEL RAY AND GARETH WILLIAMS
}

\begin{abstract}
We apply results of Harada, Holm and Henriques to prove that the Atiyah-Segal equivariant complex $K$-theory ring of a divisive weighted projective space (which is singular for nontrivial weights) is isomorphic to the ring of integral piecewise Laurent polynomials on the associated fan. Analogues of this description hold for other complex-oriented equivariant cohomology theories, as we confirm in the case of homotopical complex cobordism, which is the universal example. We also prove that the Borel versions of the equivariant $K$-theory and complex cobordism rings of more general singular toric varieties, namely those whose integral cohomology is concentrated in even dimensions, are isomorphic to rings of appropriate piecewise formal power series. Finally, we confirm the corresponding descriptions for any smooth, compact, projective toric variety, and rewrite them in a face ring context. In many cases our results agree with those of Vezzosi and Vistoli for algebraic $K$-theory, Anderson and Payne for operational $K$-theory, Krishna and Uma for algebraic cobordism, and Gonzalez and Karu for operational cobordism; as we proceed, we summarize the details of these coincidences.
\end{abstract}

\section{INTRODUCTION}

Throughout this work $G$ is a compact Lie group, and $G \subset Y$ a $G$-space; when $G$ is understood, we rewrite the latter as $Y$. Our aim is to investigate the $G$-equivariant complex $K$-theory and complex cobordism rings of certain special families of $Y$, for which $G$ is a torus $T^{n}$. Given the recent proliferation of $K$-theory and cobordism functors [AnPa], [C], [GoKa], [KU], it is important to specify precisely which we use, and to comment on their relationship with other versions as we proceed. Our underlying philosophy is closest to algebraic topology and homotopy theory.

So far as $K$-theory is concerned, we focus mainly on the unreduced Atiyah-Segal $G$-equivariant ring $K_{G}^{*}(Y)$ [Se], graded over the integers for later convenience. If $Y$ is compact, then $K_{G}^{0}(Y)$ is constructed from equivalence classes of $G$-equivariant complex vector bundles; otherwise, it is given by equivariant homotopy classes $\left[Y, \operatorname{Fred}\left(\mathcal{H}_{G}\right)\right]_{G}$, where $\mathcal{H}_{G}$ is a Hilbert space containing infinitely many copies of each irreducible representation of $G$ [AS2]. For the 1-point space $*$ with trivial $G$-action, we write the coefficient ring $K_{G}^{*}(*)$ as $K_{G}^{*}$. It is isomorphic to $R(G)\left[z, z^{-1}\right]$, where $R(G)$ denotes the complex representation ring of $G$, and realises $K_{G}^{0}$; the Bott periodicity element $z$ has cohomological dimension -2 . The equivariant projection $Y \rightarrow *$ induces the structure of a graded $K_{G}^{*}$-algebra on $K_{G}^{*}(Y)$, for any $G \odot Y$.

For complex cobordism, our primary interest is tom Dieck's $G$-equivariant $\operatorname{ring} M U_{G}^{*}(Y)$ [tD], defined by equivariant stable homotopy classes $\left[Y, M U_{G}\right]_{G}$ of maps into the Thom spectrum $M U_{G}$. Although the coefficient ring $M U_{G}^{*}$ remains undetermined, considerable information is available when $G=T^{n}$; Sinha [Si], for example, has made extensive calculations, and solved the case $n=1$. The equivariant projection $Y \rightarrow *$ induces the structure of a graded $M U_{G}^{*}$-algebra on $M U_{G}^{*}(Y)$, for any $G \subset Y$. In fact $M U_{G}^{*}(-)$ is universal amongst unreduced complex-oriented $G$ equivariant cohomology theories, at least for abelian $G$ [CGK]. The most natural link between cobordism and $K$-theory arises within this framework, and is provided by the equivariant Todd genus $t d: M U_{G}^{*}(Y) \rightarrow K_{G}^{*}(Y)[\mathrm{Ok}]$.

2010 Mathematics Subject Classification. Primary: 55N91; Secondary: 14M25, 57R18.

Key words and phrases. Divisive weight vector, Equivariant K-theory, fan, piecewise Laurent polynomial, weighted projective space. 
Given the universality of $M U_{G}^{*}(-)$, we may follow the lead of [HaHeHo] and consider arbitrary complex-oriented cohomology theories $E_{G}^{*}(-)$, although we restrict attention to cases for which $G$ is a compact torus. We view the complex orientation as a choice of equivariant Thom class without further comment. In these situations, readers will lose little by interpreting $E$ as whichever of complex $K$-theory, complex cobordism, or integral cohomology takes their fancy. When more detail is required, we shall treat $K$-theory first and cobordism second; both depend on the more familiar case $H_{G}^{*}(-)$ of integral cohomology, which we recall as necessary.

If $Y$ is an $n$-dimensional toric variety [Fu], it is automatically endowed with an action of $T^{n}$. The problem of describing the ring $K_{T^{n}}^{*}(Y)$ has already been addressed, albeit indirectly, in the symplectic context $[\mathrm{HaL}]$. From the algebraic viewpoint, however, it is more natural to study algebraic vector bundles over $Y$, and to compute the corresponding algebraic $K$-theory. Influential contributions along these lines include Brion [B], Dupont [Du], Kaneyama [Kan], Klyachko [Kl], Liu and Yao [LY], Morelli [Mor], and Payne [P], although the work of Vezzosi and Vistoli [VV] is closest to ours in spirit, and leads to answers that are isomorphic to $K_{T^{n}}^{*}(Y)$ for all smooth $Y$. The results of $[\mathrm{VV}]$ are also cited in the appendix to [RKR], with appeal to arguments of Franz. The same arguments are likely to provide an alternative approach to our own results, but have yet to be fully documented. We emphasise our insistence on integer coefficients, bearing in mind that several of the above authors tensor their $K$-groups with $\mathbb{Q}$ or $\mathbb{C}$ throughout.

The techniques used by symplectic geometers apply to a wider class of $T^{n}$-spaces, and are based on symplectic reduction, Morse theory [HoM], and GKM graphs [GKM]. Algebraic geometers, on the other hand, tend to restrict attention (at least over $\mathbb{Z}$ ) to smooth toric varieties $Y$, possibly non-compact, and express their invariants in terms of the underlying fan $\Sigma_{Y}$.

Our aim is to combine features of each viewpoint, and describe $K_{T^{n}}^{*}(Y)$ in terms of $\Sigma_{Y}$ for a certain family of singular toric varieties. Following [BFR2], we refer to these as divisive weighted projective spaces, and denote them by $\mathbb{P}(\chi)$. Recent work [BFNR, Theorem 1.2] shows that any weighted projective space is homotopy equivalent to one which is divisive, but such equivalences need not be equivariant, by the concluding remarks of [BFR1, Section 5].

We are motivated by related calculations of the Borel equivariant cohomology of more general singular examples, in which $H_{T^{n}}^{*}(Y)$ is identified with the graded ring of piecewise polynomials on $\Sigma_{Y}$ [BFR1]. In its simplest form, our main result states the following.

Theorem 1.1. For any divisive weighted projective space, $K_{T^{n}}^{0}(\mathbb{P}(\chi))$ is isomorphic as a $K_{T^{n}}^{0}$ algebra to the ring of piecewise Laurent polynomials on $\Sigma_{Y}$; furthermore, $K_{T^{n}}^{1}(\mathbb{P}(\chi))$ is zero.

A precise statement is proven as Theorem 5.5.1 in Section 5.

More recently, Anderson and Payne [AnPa] have introduced equivariant operational algebraic $K$-theory, and identified the rings op $K_{T^{n}}^{\circ}(Y)$ with piecewise Laurent polynomials on $\Sigma_{Y}$. Their calculations are valid for all toric varieties, and therefore agree with ours on divisive weighted projective spaces.

Turning to $M U_{T^{n}}^{*}(\mathbb{P}(\chi))$, we note first that algebraic geometers have developed a successful theory of algebraic cobordism during the last 15 years, by working over the Lazard $\operatorname{ring} L^{*}$. They have also introduced equivariant versions that are related to $M U_{T^{n}}^{*}(-)$. As described by Krishna and Uma $[\mathrm{KU}]$, for example, these theories are complete; so their coefficient rings cannot be isomorphic to $M U_{T^{n}}^{*}$. Nevertheless, the equivariant algebraic cobordism ring of many toric varieties $Y$ may be expressed in terms of piecewise formal power series on $\Sigma_{Y}$ [KU]. As we explain below, this is isomorphic to the Borel equivariant cobordism ring $M U^{*}\left(E T^{n} \times_{T^{n}} Y\right)$ in cases such as smooth $Y$, or products of weighted projective spaces.

Our conclusions for complex cobordism are based on the fact that $M U_{T^{n}}^{*}$ is an algebra over the Lazard ring $L^{*}$, graded cohomologically. So we refer to $M U_{T^{n}}^{*}$ as the ring of $T^{n}$-cobordism forms, and express our second result accordingly. 
Theorem 1.2. For any divisive weighted projective space, $M U_{T^{n}}^{*}(\mathbb{P}(\chi))$ is isomorphic as an $M U_{T^{n}}^{*}$ algebra to the ring of piecewise cobordism forms on $\Sigma_{Y}$; in particular, $M_{T^{n}}^{*}(\mathbb{P}(\chi)$ ) is zero in odd dimensions.

A precise statement is proven as Theorem 5.5.2 in Section 5.

Most recently, inspired by [AnPa], Gonzalez and Karu [GoKa] have defined equivariant operational algebraic cobordism. For any quasiprojective toric variety $Y$, they identify their operational ring with the ring of piecewise formal power series on $\Sigma_{Y}$, and therefore with $M U^{*}\left(E T^{n} \times_{T^{n}} Y\right)$ for smooth $Y$, or products of weighted projective spaces.

We introduce weighted projective spaces as singular toric varieties in Section 2, focusing on divisive examples $\mathbb{P}(\chi)$ and their invariant $\mathrm{CW}$-structures. In Section 3 we recall the generalised GKM-theory that allows us to compute $K_{T^{n}}^{*}(Y)$ and $M U_{T^{n}}^{*}(Y)$ for certain stratified $T^{n}$-spaces $Y$, and confirm that the theory applies to $\mathbb{P}(\chi)$. In order to rewrite the outcome in the language of Theorems 1.1 and 1.2, we devote Section 4 to describing diagrams of algebras, and piecewise structures on arbitrary fans. We combine the two viewpoints in Section 5, and deduce a version of Theorems 1.1 and 1.2 that holds for a wider class of equivariant cohomology theories. In Section 6 we relate $K_{T^{n}}^{*}(\mathbb{P}(\chi))$ and $M U_{T^{n}}^{*}(\mathbb{P}(\chi))$ to the Borel equivariant $K$-theory and cobordism of $\mathbb{P}(\chi)$, in terms of piecewise formal power series, the Chern character, and the Boardman homomorphism. Finally, in Section 7, we extend our conclusions to smooth toric varieties, and rewrite the resulting piecewise algebras in the context of face rings.

Before we begin, it is convenient to introduce notation and conventions that we shall use without further comment.

We write $S^{1}$ for the circle as a topological space, and $T<\mathbb{C}^{\times}$for its realisation as the group of unimodular complex numbers under multiplication. The $(n+1)$-dimensional compact torus $T^{n+1}$ is a subgroup of the algebraic torus $\left(\mathbb{C}^{\times}\right)^{n+1}$, and acts on $\mathbb{C}^{n+1}$ by coordinate-wise multiplication. This is the standard action; it preserves the unit sphere $S^{2 n+1} \subset \mathbb{C}^{n+1}$, and the corresponding orbit space may be identified with the standard $n$-simplex $\Delta^{n} \subset \mathbb{R}_{\geqslant}^{n+1}$ in the positive orthant.

Readers who require background information and further references on equivariant topology may consult $[\mathrm{AlPu}]$, and the survey articles in [Ma]. For fans, toric varieties, and their topological aspects, we suggest $[\mathrm{Fr}],[\mathrm{Fu}]$ and $[\mathrm{Od}]$.

Acknowledgments. We are especially grateful to Dave Anderson and Sam Payne for their advice and encouragement on matters of algebraic geometry, to Tony Bahri and Matthias Franz for many hours of discussion on the topology of weighted projective spaces, and to the Hausdorff Institute in Bonn for supporting our work together on this and related projects. Megumi Harada was partially supported by an Early Researcher Award from the Ministry of Research and Innovation of Ontario, and a Discovery Grant from the NSERC of Canada; Tara Holm was partially supported by a PCCW Affinito-Stewart Grant, Grant 208975 from the Simons Foundation, and NSF Grant DMS-1206466.

\section{Weighted PRojective SPACE}

Weighted projective spaces are amongst the simplest and most elegant examples of toric orbifolds $[\mathrm{Fu}]$, and we devote this section to summarising their definition and basic properties.

A weight vector $\chi$ is a sequence $\left(\chi_{0}, \ldots, \chi_{n}\right)$ of $n+1$ positive integers; $\chi$ determines a subcircle $T(\chi)<T^{n+1}$ by

$$
T(\chi)=\left\{\left(t^{\chi_{0}}, \ldots, t^{\chi_{n}}\right):|t|=1\right\}
$$

which acts on $S^{2 n+1}$ with finite stabilizers. Then the weighted projective space $\mathbb{P}(\chi)$ is defined to be the orbit space $S^{2 n+1} / T(\chi)$. Each point of $\mathbb{P}(\chi)$ may be written as an equivalence class $[z]=\left[z_{0}, \ldots, z_{n}\right]$, where the $z_{j}$ are known as homogenous coordinates. Permutations of the $z_{j}$ induce self-homeomorphisms of $\mathbb{P}(\chi)$, so we may reorder the weights as required; it is often convenient to 
assume that they are non-decreasing. Of course $\mathbb{P}(\chi)$ may equally well be exhibited as the orbit space $\left(\mathbb{C}^{n+1} \backslash\{0\}\right) / \mathbb{C}^{\times}(\chi)$, and therefore as a complex algebraic variety.

The finite stabilisers ensure that $\mathbb{P}(\chi)$ is an orbifold, which is singular for $n>1$ unless $\chi=$ $(d, \ldots, d)$ for some positive integer $d$. The residual action of the torus $T^{n} \cong T^{n+1} / T(\chi)$ turns $\mathbb{P}(\chi)$ into a toric orbifold, with quotient polytope an $n$-simplex. If $\chi=(1, \ldots, 1)$, then $T(\chi)$ is the diagonal circle $T_{\delta}<T^{n+1}$, and $\mathbb{P}(\chi)$ reduces to the standard projective space $\mathbb{C} P^{n}$. In this case, $K_{T^{n}}^{*}\left(\mathbb{C} P^{n}\right)$ is computed in $[\mathrm{GW}, \S 4.1]$.

Orbifolds may be studied from a number of different perspectives, and recent articles have focused on their interpretation as groupoids [Moe] and as stacks [L]. Several invariants of these richer structures have been defined, such as the orbifold fundamental group [ALR]. Nevertheless, in this work we remain firmly in the topological world, and study the underlying topological space $\mathbb{P}(\chi)$; in other contexts, it is known as the coarse moduli space of the stack. There has been a recent surge of interest [BFR1], [BFNR] in its $T^{n}$-equivariant topological invariants.

By construction, $\mathbb{P}(d \chi)$ is equivariantly homeomorphic to $\mathbb{P}(\chi)$ for any positive integer $d$, although they differ as orbifolds. For our purposes, it therefore suffices to assume that the greatest common divisor $\operatorname{gcd}(\chi)$ is 1 ; in orbifold terminology, this is tantamount to restricting attention to effective cases. If $\operatorname{gcd}(\chi)=1$, then [Do] provides an equivariant homeomorphism

$$
\mathbb{P}\left(d \chi_{0}, \ldots, d \chi_{j-1}, \chi_{j}, d \chi_{j+1}, \ldots, d \chi_{n}\right) \cong \mathbb{P}(\chi)
$$

for any $0 \leq j \leq n$, and any positive integer $d$ such that $\operatorname{gcd}\left(d, \chi_{j}\right)=1$. Further simplification is therefore possible by insisting that $\chi$ be normalised, in the sense that

$$
\operatorname{gcd}\left(\chi_{0}, \ldots, \widehat{\chi_{j}}, \ldots, \chi_{n}\right)=1
$$

for every $0 \leq j \leq n$.

We may impose additional restrictions on the weights, with important topological consequences.

Definitions 2.2. The weight vector $\chi$ and the weighted projective space $\mathbb{P}(\chi)$ are

1. weakly divisive if $\chi_{j}$ divides $\chi_{n}$ for every $0 \leq j<n$,

2. divisive if $\chi_{j-1}$ divides $\chi_{j}$ for every $1 \leq j \leq n$.

A divisive $\chi$ is automatically weakly divisive, and is necessarily non-decreasing. Moreover, $\chi$ is divisive precisely when the reverse sequence $\chi_{n}, \ldots, \chi_{0}$ is well ordered, in the sense of Nishimura and Yosimura $[\mathrm{NY}]$.

Remark 2.3 ([BFR2, Theorem 3.7, Corollary 3.8]). If $\mathbb{P}(\chi)$ is weakly divisive, then it is homeomorphic to the Thom space of a complex line bundle over $\mathbb{P}\left(\chi^{\prime}\right)$, where $\chi^{\prime}=\left(\chi_{0}, \ldots, \chi_{n-1}\right)$; if it is divisive, then it is homeomorphic to an $n$-fold iterated Thom space of complex line bundles over the one-point space $*$.

In case $\chi_{0}=1$, there exists a canonical isomorphism $j: T^{n} \rightarrow T^{n+1} / T(\chi)$, defined by setting $j\left(u_{1}, \ldots, u_{n}\right)=\left[1, u_{1}, \ldots, u_{n}\right]$; the resulting action of $T^{n}$ on $\mathbb{P}(\chi)$ satisfies

$$
\left(u_{1}, \ldots, u_{n}\right) \cdot\left[z_{0}, z_{1}, \ldots, z_{n}\right]=\left[z_{0}, u_{1} z_{1}, \ldots, u_{n} z_{n}\right] .
$$

From this point onwards, we therefore make the following assumptions.

\section{Assumptions 2.5.}

1. The weight vector $\chi$ is both normalised and divisive, so $\chi_{0}=\chi_{1}=1$.

2. The residual action $T^{n} \odot \mathbb{P}(\chi)$ is given by the isomorphism $j$ and (2.4).

For any weighted projective space, Kawasaki's calculations [Kaw] imply the existence of a homotopy equivalent $\mathrm{CW}$-complex with a single cell in every even dimension. This has been realized in [BFNR], but current evidence suggests that an explicit cellular decomposition for the general case 
is unpleasantly complicated [ON]. Nevertheless, Remark 2.3 provides an easy solution for divisive $\chi$. Given any $1 \leq i \leq n$, it is convenient to write $D^{2 i}$ for the closed unit disc

$$
\left\{w:\left|w_{n-i+1}\right|^{2}+\cdots+\left|w_{n}\right|^{2} \leq 1\right\} \subset \mathbb{C}^{i},
$$

and $g_{i}: D^{2 i} \rightarrow \mathbb{P}(\chi)$ for the map given by

$$
g_{i}(w)=\left[0, \ldots, 0,\left(1-\left|w_{n-i+1}\right|^{2}-\cdots-\left|w_{n}\right|^{2}\right)^{1 / 2}, w_{n-i+1}, \ldots, w_{n}\right] .
$$

For $i=0$, let $D^{0}=\{0\}$ and $g_{0}(0)=[0, \ldots, 0,1]$.

Proposition 2.7. For every divisive $\mathbb{P}(\chi)$, the $g_{i}$ are characteristic maps for a $C W$-structure that contains exactly $n+1$ cells.

Proof. For $1 \leq i \leq n$, the restriction of $g_{i}$ to the interior of $D^{2 i}$ is a homeomorphism onto

$$
\left\{[z]: z_{0}=\cdots=z_{n-i-1}=0, z_{n-i} \neq 0\right\} \subset \mathbb{P}(\chi),
$$

which is therefore an open $2 i$-cell. Furthermore, $g_{i}$ maps the boundary of $D^{2 i}$ onto the subspace $\left\{[z]: z_{0}=\cdots=z_{n-i}=0\right\}$, which is the union of all lower dimensional cells. The zero cell is $\{[0, \ldots, 0,1]\}$.

Corollary 2.9. The $C W$-structure is invariant under the residual action of $T^{n}$.

Proof. The action (2.4) automatically preserves the conditions of (2.8).

Combining (2.4) and (2.6) shows that the characteristic map $g_{i}$ induces the action

$$
\left(u_{1}, \ldots, u_{n}\right) \cdot\left(w_{n-i+1}, \ldots, w_{n}\right)=\left(u_{n-i+1} u_{n-i}^{-\chi_{n-i+1} / \chi_{n-i}} w_{n-i+1}, \ldots, u_{n} u_{n-i}^{-\chi_{n} / \chi_{n-i}} w_{n}\right)
$$

of $T^{n}$ on $D^{2 i}$, for each $1 \leq i \leq n$ (taking $u_{0}=1$ in case $i=n$ ). This is the unit disc $D\left(\rho_{i}\right)$ of an $i$-dimensional unitary representation $\rho_{i}$ of $T^{n}$.

We denote the CW-structure of Proposition 2.7 by $\mathbb{P}(\chi)=e^{0} \cup e^{2} \cup \cdots \cup e^{2 n}$, where $e^{2 i}$ is the closure of $(2.8)$ in $\mathbb{P}(\chi)$; the centers $[0, \ldots, 0,1,0, \ldots, 0]$ of the cells are precisely the fixed points of the residual action.

\section{Generalized GKM-Theory}

In this section we recall the generalized GKM-theory of [HaHeHo], and explain its application to Corollary 2.9. This leads to a description of $E_{T^{n}}^{*}(\mathbb{P}(\chi))$ for divisive $\chi$ and several examples of $E_{T^{n}}$, including equivariant complex $K$-theory and cobordism.

Following [HaHeHo, §3], we require the space $G \subset Y$ to be equipped with a $G$-invariant stratification $Y=\bigcup_{i \in I} Y_{i}$, and write $Y_{<i}$ for the subspace $\bigcup_{j<i} Y_{j} \subset Y_{i}$ for every $i \in I$. We insist that each $Y_{i}$ contains a subspace $F_{i}$, whose neighbourhood is homeomorphic to the total space $V_{i}$ of a

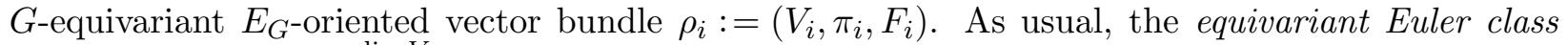
$e_{G}\left(\rho_{i}\right)$ is defined in $E_{G}^{\operatorname{dim} V_{i}}\left(F_{i}\right)$ by restricting the chosen equivariant Thom class of $\rho_{i}$ to the zero section, for every $i \in I$.

We recall the following four assumptions of [HaHeHo], which insure that $E_{G}^{*}(Y)$ may be computed by their methods. As we shall see, they are satisfied by every divisive $\mathbb{P}(\chi)$.

\section{Assumptions 3.1.}

1. Each subquotient $Y_{i} / Y_{<i}$ is homeomorphic to the Thom space Th $\left(\rho_{i}\right)$, with attaching maps $\varphi_{i}: S\left(\rho_{i}\right) \rightarrow Y_{<i}$.

2. Every $\rho_{i}$ admits a $G$-equivariant direct sum decomposition $\bigoplus_{j<i} \rho_{i, j}$ into $E_{G}$-oriented subbundles $\rho_{i, j}=\left(V_{i, j}, \pi_{i, j}, F_{i}\right)$.

3. There exist $G$-equivariant maps $f_{i, j}: F_{i} \rightarrow F_{j}$ such that the restrictions $\left.f_{i, j} \circ \pi_{i, j}\right|_{S\left(\rho_{i, j}\right)}$ and $\left.\varphi_{i}\right|_{S\left(\rho_{i, j}\right)}$ agree for every $j<i$. 
4. The Euler classes $e_{G}\left(\rho_{i, j}\right)$ are not divisors of zero in $E_{G}^{*}\left(F_{i}\right)$ for any $j<i$, and are pairwise relatively prime.

Note that the $\rho_{i, j}$ may have dimension 0. Assumption 3.1.4 means that $e_{G}\left(\rho_{i, j}\right)$ divides $y$ for each $j$ if and only if $e_{G}\left(\rho_{i}\right)$ divides $y$, for any $y \in E_{G}^{*}\left(F_{i}\right)$.

Now let $\iota^{*}: E_{G}^{*}(Y) \rightarrow \prod_{i} E_{G}^{*}\left(F_{i}\right)$ be the homomorphism induced by the inclusion $\coprod_{i} F_{i} \subset Y$.

Theorem 3.2 ([HaHeHo, Theorem 3.1]). Let $Y$ be a G-space satisfying the four Assumptions 3.1; then $\iota^{*}$ is monic, and has image

$$
\Gamma_{Y}:=\left\{y=\left(y_{i}\right): e_{G}\left(\rho_{i, j}\right) \text { divides } y_{i}-f_{i, j}^{*}\left(y_{j}\right) \text { for all } j<i\right\} \leq \prod_{i} E_{G}^{*}\left(F_{i}\right) .
$$

As in several of the examples in [HaHeHo], our application to Corollary 2.9 involves a $T^{n_{-}}$ invariant skeletal filtration. Specifically, $Y_{i}=\cup_{j \leq i} e^{2 j}$ is the $2 i$-skeleton of $\mathbb{P}(\chi)$ for $0 \leq i \leq n$, and the $F_{i} \subset Y_{i}$ contain only the centers of the cells $e^{2 i}$. These are the fixed points of the $T^{n}$-action, and the $T^{n}$-equivariant bundles $\rho_{i}$ reduce to $i$-dimensional complex representations, which are

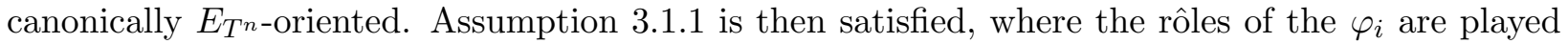
by the restrictions of the $g_{i}$ of Proposition 2.7 to $S^{2 i-1}$. The equivariant Euler classes $e_{T^{n}}\left(\rho_{i}\right)$ lie in the coefficient ring $E_{T^{n}}^{*}$.

In order to check Assumption 3.1.2, we refer back to (2.10). Each $\rho_{i}$ decomposes as a sum $\bigoplus_{j<i} \rho_{i, j}$ of 1 -dimensionals, where $\rho_{i, j}$ is defined by

$$
\left(u_{1}, \ldots, u_{n}\right) \cdot w_{n-j}=u_{n-j} u_{n-i}^{-\chi_{n-j} / \chi_{n-i}} w_{n-j}
$$

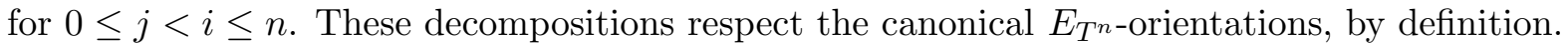

For Assumption 3.1.3, the maps $f_{i, j}$ are necessarily constant and equivariant, so the restrictions to $S\left(\rho_{i, j}\right)$ of $f_{i, j} \circ \pi_{i, j}$ and $g_{i}$ agree, for every $j<i$.

Before confirming Assumption 3.1.4, recall $[\mathrm{Hu}]$ that the complex representation ring of $T^{n}$ is isomorphic to the Laurent polynomial algebra

$$
R\left(T^{n}\right) \cong S_{\mathbb{Z}}^{ \pm}(\alpha):=\mathbb{Z}\left[\alpha_{1}, \ldots, \alpha_{n}\right]_{\left(\alpha_{1} \cdots \alpha_{n}\right)}
$$

on generators $\alpha_{j}$, which represent the 1-dimensional irreducibles defined by projection onto the $j$ th coordinate circle. In particular, (3.3) states that

$$
\rho_{i, j} \cong \alpha_{n-j} \alpha_{n-i}^{-\chi_{n-j} / \chi_{n-i}}
$$

(taking $\alpha_{0}=1$ in case $i=n$ ). Since equivariant Euler classes behave exponentially, $e_{T^{n}}$ is determined on any representation by its value on the monomials $\alpha^{J}:=\alpha_{1}^{j_{1}} \cdots \alpha_{n}^{j_{n}}$, which form an additive basis for $R\left(T^{n}\right)$ as $J$ ranges over $\mathbb{Z}^{n}$.

In the case of $K$-theory, the coefficient ring $K_{T^{n}}^{*}$ is isomorphic to $R\left(T^{n}\right)$ in even dimensions, and is zero in odd [Se]. Periodicity may be made explicit by incorporating the Bott element $z$ into (3.4) and writing

$$
K_{T^{n}}^{*} \cong S_{K^{*}}^{ \pm}(\alpha) \cong R\left(T^{n}\right)\left[z, z^{-1}\right],
$$

where $\alpha^{J}$ (for any $J$ ) and $z$ have cohomological dimensions 0 and -2 respectively. The notation reflects the fact that the coefficient ring $K^{*}$ is isomorphic to $\mathbb{Z}\left[z, z^{-1}\right]$. Then $e_{T^{n}}\left(\alpha^{J}\right)=1-\alpha^{ \pm J}$, where both choices of sign occur in the literature. Some authors even prefer $z^{-1}\left(1-\alpha^{ \pm J}\right)$, to achieve greater consistency with cobordism and cohomology by realizing the Euler class in cohomological dimension 2 ; here, for notational convenience, we employ $1-\alpha^{J}$. The kernel of the augmentation $K_{T^{n}}^{*} \rightarrow K^{*}$ is the ideal $\left(1-\alpha_{1}, \ldots, 1-\alpha_{n}\right)$.

In the case of complex cobordism, the coefficient ring $M U_{T^{n}}^{*}$ is an algebra over $L^{*}$, and is freely generated as an $L^{*}$-module by even-dimensional elements [C]. The Euler classes $e_{T^{n}}\left(\alpha^{J}\right)$ are nonzero elements of $M U_{T^{n}}^{2}$, and are denoted by $e\left(\alpha^{J}\right)$ in the calculations of [Si] and elsewhere; they 
generate the positive-dimensional subring $M U_{T^{n}}^{>0}$. The kernel of the augmentation $M U_{T^{n}}^{*} \rightarrow L^{*}$ is the ideal $\left(e\left(\alpha_{1}\right), \ldots, e\left(\alpha_{n}\right)\right)[\mathrm{CM}]$.

In the case of Borel equivariant integral cohomology, the coefficient ring $H_{T^{n}}^{*}$ is isomorphic to the polynomial algebra

$$
H^{*}\left(B T^{n} ; \mathbb{Z}\right) \cong S_{\mathbb{Z}}(x):=\mathbb{Z}\left[x_{1}, \ldots, x_{n}\right]
$$

on 2-dimensional generators $x_{j}$. Then $e_{T^{n}}\left(\alpha^{J}\right)=\sum_{J} j_{k} x_{k}$ for any $J$; in particular, the equation $e_{T^{n}}\left(\alpha_{i}\right)=x_{i}$ may be taken to define $x_{i}$ for every $1 \leq i \leq n$. The kernel of of the augmentation $H_{T^{n}}^{*} \rightarrow H^{*}$ is the ideal $\left(x_{1}, \ldots, x_{n}\right)$.

So from (3.5), we deduce that

$$
e_{T^{n}}\left(\rho_{i, j}\right)=\left\{\begin{array}{l}
1-\alpha_{n-j} \alpha_{n-i}^{-\chi_{n-j} / \chi_{n-i}} \text { in } K_{T^{n}}^{0} \\
e\left(\alpha_{n-j} \alpha_{n-i}^{-\chi_{n-j} / \chi_{n-i}}\right) \text { in } M U_{T^{n}}^{2} \\
x_{n-j}-\left(\chi_{n-j} / \chi_{n-i}\right) x_{n-i} \text { in } H_{T^{n}}^{2}
\end{array}\right.
$$

for $0 \leq j<i \leq n$.

In each of these three cases, the ambient ring is an integral domain; for $M U_{T^{n}}^{*}$, this is proven in [Si, Corollary 5.3]. So none of the Euler classes of (3.8) are divisors of zero. The following criteria address the remaining parts of Assumption 3.1.4.

Criteria 3.9 ([HaHeHo, Lemma 5.2]). Given any finite set of non-zero $\alpha^{J}$, their equivariant Euler classes are pairwise relatively prime in $K_{T^{n}}^{*}$ or $M U_{T^{n}}^{*}$ whenever no two $J$ are linearly dependent in $\mathbb{Z}^{n}$; the additional condition that no prime $p$ divides any two $J$ is required in $H_{T^{n}}^{*}$.

For $\rho_{i, j}$ with $i<n,(3.5)$ shows that $J$ has only two non-zero entries, namely 1 in position $n-j$ and $-\chi_{n-j} / \chi_{n-i}$ in position $n-i$; for $\rho_{n, j}$, there is a single 1 in position $n-j$. So Criteria 3.9 confirm that the Euler classes $e_{T^{n}}\left(\rho_{i, j}\right)$ are pairwise relatively prime in all three cases, and therefore that Assumption 3.1.4 also holds.

We may now conclude our first description of $E_{T^{n}}^{*}(\mathbb{P}(\chi))$.

Proposition 3.10. For any divisive weighted projective space, $E_{T^{n}}^{*}(\mathbb{P}(\chi))$ is isomorphic as an $E_{T^{n}}^{*}$-algebra to the subring

$$
\Gamma_{\mathbb{P}(\chi)}=\left\{y: e_{T^{n}}\left(\rho_{i, j}\right) \text { divides } y_{i}-y_{j} \text { for all } j<i\right\} \leq \prod_{i} E_{T^{n}}^{*},
$$

in each of the cases $E=K, M U, H$.

Proof. Our preceding analysis shows that Theorem 3.2 applies directly to the skeletal filtration. Compatibility with the $E_{T^{n}}^{*}$-algebra structure follows immediately.

Proposition 3.10 shows that $E_{T^{n}}^{*}(\mathbb{P}(\chi))$ is zero in odd dimensions.

The idea behind Theorem 5.5 is to convert Proposition 3.10 into a form more directly related to the properties of the fan $\Sigma_{\chi}:=\Sigma_{\mathbb{P}(\chi)}$.

\section{Piecewise Algebra}

Before stating Theorem 5.5, we introduce certain algebraic and geometric constructions that are associated to fans by the theory of diagrams. They are motivated by modern approaches to homotopy theory, and provide a common language in which to address the cases under discussion.

A rational fan $\Sigma$ in $\mathbb{R}^{n}$ determines a small category $\operatorname{CAT}(\Sigma)$, whose objects are the cones $\sigma$ and morphisms their inclusions $i_{\sigma, \tau}: \sigma \subseteq \tau$. The zero cone $\{0\}$ is initial, and the maximal cones admit only identity morphisms. The opposite category $\operatorname{CAT}^{o p}(\Sigma)$ has morphisms $p_{\tau, \sigma}: \tau \supseteq \sigma$, and $\{0\}$ is final.

For $0 \leq d \leq n$, the set of $d$-dimensional cones is denoted by $\Sigma(d) \subseteq \Sigma$. The elements of $\Sigma(1)$ are known as rays, and are represented by primitive vectors $v_{1}, \ldots, v_{m}$, where $m$ denotes the 
cardinality of $\Sigma(1)$ henceforth. Each cone may be identified by its generating rays $v_{j_{1}}, \ldots, v_{j_{k}}$, and interpreted as a subset $\sigma \subseteq \Sigma(1)$. The cardinality $k=|\sigma|$ coincides with the dimension $d=\operatorname{dim}(\sigma)$ if and only if the cone $\sigma$ is simplicial.

Every $d$-dimensional $\sigma$ gives rise to an $(n-d)$-dimensional subspace $\mathbb{R}_{\sigma^{\perp}} \subseteq \mathbb{R}^{n}$, by forming the orthogonal complement of its linear hull $\mathbb{R}_{\sigma}$. The rationality of $\sigma$ implies that $\mathbb{R}_{\sigma \perp} \cap \mathbb{Z}^{n}$ has rank $(n-d)$, and admits a basis $w_{1}, \ldots, w_{n-d}$ of integral vectors; it is unique up to the action of $G L(n-d, \mathbb{Z})$, and determines the linear forms

$$
w_{c}^{t r} x=w_{c, 1} x_{1}+\cdots+w_{c, n} x_{n} \text { for } 1 \leq c \leq n-d .
$$

The intersection of their kernels is $\mathbb{R}_{\sigma}$, and there exists a splitting $\mathbb{R}^{n} \cong \mathbb{R}_{\sigma^{\perp}} \times \mathbb{R}_{\sigma}$. It is convenient to interpret $\mathbb{R}^{n}$ as the Lie algebra of $T^{n}$ and write the associated splitting as

$$
T^{n} \cong T_{\sigma^{\perp}} \times T_{\sigma}
$$

where the Lie algebra of $T_{\sigma^{\perp}}$ is spanned by the $w_{c}$, for any cone $\sigma$. Thus $T_{\sigma^{\perp}}=\{1\}$ for topdimensional cones, and $T_{\{0\}^{\perp}}=T^{n}$.

Definitions 4.3. A $\Sigma$-diagram in a category C is a covariant functor $F$ : CAT $(\Sigma) \rightarrow$ C; similarly, a $\Sigma^{o p}$-diagram (or contravariant $\Sigma$-diagram) is a covariant functor $F: \mathrm{CAT}^{o p}(\Sigma) \rightarrow \mathrm{C}$.

We are interested in diagrams for which one or both of $\lim F$ and colim $F$ exist in C.

Definitions 4.3 are motivated by a familiar diagram in TOP, which underlies the construction of the toric variety $X_{\Sigma}$ as a topological $T^{n}$-space. It is denoted by $U: \operatorname{CAT}(\Sigma) \rightarrow T^{n}$-TOP, and uses the dual cones $\sigma^{\vee}$ in the lattice $M:=\left(\mathbb{Z}^{n}\right)^{\vee}$; it is given by

$$
U(\sigma)=U_{\sigma}:=\operatorname{Hom}\left(\sigma^{\vee} \cap M, \mathbb{C}^{\times} \cup\{0\}\right) \text { and } U\left(i_{\sigma, \tau}\right)=j_{\sigma, \tau},
$$

where $\operatorname{Hom}(-)$ denotes the affine variety of semigroup homomorphisms, and $j_{\sigma, \tau}: U_{\sigma} \rightarrow U_{\tau}$ is induced by $i_{\sigma, \tau}^{\vee}: \tau^{\vee} \rightarrow \sigma^{\vee}$. The standard description of $X_{\Sigma}$, as given by [Fu, §1.4], for example, may then be expressed as the colimit $\operatorname{colim} U$ in $T^{n}$-TOP.

In fact $T^{n} \odot U_{\sigma}$ is $T^{n}$-equivariantly homotopy equivalent to $T^{n} \odot T^{n} / T_{\sigma}$ for every cone $\sigma$ [CLS, Proposition 12.1.9, Lemma 3.2.5]. So $U$ is objectwise equivariantly equivalent to the diagram

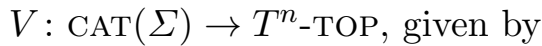

$$
V(\sigma)=T^{n} / T_{\sigma} \text { and } V\left(i_{\sigma, \tau}\right)=r_{\sigma, \tau},
$$

where $r_{\sigma, \tau}$ is the projection induced by the inclusion $T_{\sigma} \leq T_{\tau}$. Since $U$ is cofibrant, it follows that hocolim $V$ is equivariantly homotopy equivalent to $\operatorname{colim} U=X_{\Sigma}$. Diagram (4.5) first appeared in [WZZ], and more recently in [Fr].

We may now describe our basic examples of $\Sigma^{o p}$-diagrams in the category GCALG $_{E}$ of graded commutative $E_{T^{n}}^{*}$-algebras.

Definition 4.6. For any complex-oriented equivariant cohomology theory $E_{T^{n}}^{*}(-)$, the diagram $E V: \operatorname{CAT}^{o p}(\Sigma) \rightarrow \mathrm{GCALG}_{E}$ has

$$
E V(\sigma)=E_{T^{n}}^{*}\left(T^{n} / T_{\sigma}\right) \text { and } E V\left(p_{\tau, \sigma}\right)=r_{\sigma, \tau}^{*} .
$$

The limit $P_{E}(\Sigma)$ of $E V$ is the $E_{T^{n}}^{*}$-algebra of piecewise coefficients on $\Sigma$.

Remarks 4.8. By definition, $P_{E}(\Sigma)$ is an $E_{T^{n}}^{*}$-subalgebra of $\prod_{\sigma} E V(\sigma)$, so every piecewise coefficient $f$ has one component $f^{\sigma}$ for each cone $\sigma$ of $\Sigma$. If $\sigma$ is top dimensional, then $T_{\sigma}=T^{n}$ and $f^{\sigma}$ is a genuine element of $E_{T^{n}}^{*}$; on the other hand, $T_{\{0\}}=\{1\}$ and $f^{\{0\}}$ lies in $E^{*}$. The components of $f$ are compatible under the homomorphisms $j_{\tau, \sigma}^{*}$, and are congruent modulo the augmentation ideal. Sums and products of piecewise coefficients are taken conewise, and $E_{T^{n}}^{*} \leq P_{E}(\Sigma)$ occurs as the subalgebra of global coefficients, whose components agree on every cone. In particular, it contains the global constants 0 and 1 , which act as zero and unit respectively. 
In many cases, $E V$ and $P_{E}$ may be described more explicitly, as follows.

Suppose that $\rho$ has codimension 1 , and that $w_{1}$ is a primitive vector generating $\mathbb{R}_{\rho^{\perp}}$. The splitting (4.2) ensures that the natural action of $T^{n}$ on $T^{n} / T_{\rho}$ may then be identified with the unit circle $S(\eta)$ of the irreducible representation $\eta:=\alpha^{w_{1}}$, on which the circle $T_{\rho^{\perp}}$ acts freely and the $(n-1)$-torus $T_{\rho}$ acts trivially. The inclusion of $S(\eta)$ into the unit disc $D(\eta)$ determines the equivariant cofiber sequence

$$
S(\eta) \longrightarrow D(\eta) \longrightarrow S^{\eta}
$$

where $S^{\eta}$ denotes the one-point compactification $T^{n} \odot D(\eta) / S(\eta)$. Applying $E_{T^{n}}^{*}(-)$ yields the long exact sequence

$$
\cdots \longrightarrow E_{T^{n}}^{*}\left(S^{\eta}\right) \stackrel{\cdot e}{\longrightarrow} E_{T^{n}}^{*}(D(\eta)) \longrightarrow E_{T^{n}}^{*}(S(\eta)) \longrightarrow \cdots .
$$

Since $D(\eta)$ is equivariantly contractible and the Thom isomorphism applies to the Thom space $S^{\eta}$, the homomorphism $e$ may be interpreted as multiplication by the Euler class $e_{T^{n}}(\eta)$. So $e$ is monic for each of $E=K, M U, H$; thus (4.9) becomes short exact, yielding isomorphisms

$$
E_{T^{n}}^{*} /\left(e_{T^{n}}(\eta)\right) \cong E_{T^{n}}^{*}(S(\eta)) \cong E_{T^{n}}^{*}\left(T^{n} / T_{\rho}\right)=E V(\rho)
$$

of $E_{T^{n}}^{*}$-algebras.

This calculation extends to lower dimensional cones $\tau$ by iteration. If $\tau$ has dimension $k$, then the natural action of $T^{n}$ on $T^{n} / T_{\tau}$ may be identified with the product $S\left(\eta_{1}\right) \times \cdots \times S\left(\eta_{n-k}\right)$, where $\eta_{c}$ denotes the irreducible $\alpha^{w_{c}}$ for $1 \leq c \leq n-k$. The $(n-k)$-torus $T_{\tau^{\perp}}$ acts freely, and the $k$-torus $T_{\tau}$ acts trivially, yielding isomorphisms

$$
E_{T^{n}}^{*} /\left(e_{T^{n}}\left(\eta_{1}\right), \ldots, e_{T^{n}}\left(\eta_{n-k}\right)\right) \cong E_{T^{n}}^{*}\left(S\left(\eta_{1}\right) \times \cdots \times S\left(\eta_{n-k}\right)\right) \cong E_{T^{n}}^{*}\left(T^{n} / T_{\tau}\right)=E V(\tau)
$$

If $\sigma \subset \tau$ has dimension $d<k$, then $\mathbb{R}_{\sigma^{\perp}}$ arises from $\mathbb{R}_{\tau^{\perp}}$ by adjoining additional basis vectors $w_{n-k+1}, \ldots, w_{n-d}$, and the projection

$$
q_{\tau, \sigma}: E_{T^{n}}^{*} /\left(e_{T^{n}}\left(\eta_{1}\right), \ldots, e_{T^{n}}\left(\eta_{n-k}\right)\right) \longrightarrow E_{T^{n}}^{*} /\left(e_{T^{n}}\left(\eta_{1}\right), \ldots, e_{T^{n}}\left(\eta_{n-d}\right)\right)
$$

corresponds to $r_{\tau, \sigma}^{*}: E_{T^{n}}^{*}\left(T^{n} / T_{\tau}\right) \rightarrow E_{T^{n}}^{*}\left(T^{n} / T_{\sigma}\right)$ under (4.10).

We conclude that (4.7) may be rewritten as

$$
E V(\sigma)=E_{T^{n}}^{*} /\left(e_{T^{n}}\left(\eta_{1}\right), \ldots, e_{T^{n}}\left(\eta_{n-d}\right)\right) \text { and } E V\left(p_{\tau, \sigma}\right)=q_{\tau, \sigma},
$$

and proceed to describing the examples $E=K, M U, H$ in these terms.

For $E=K$, we work with graded commutative algebras over the Laurent polynomial ring $S_{K^{*}}^{ \pm}(\alpha)$ of $(3.6)$.

Example 4.12. The Laurent polynomial diagram $K V: \mathrm{CAT}^{o p}(\Sigma) \rightarrow \mathrm{GCALG}_{K}$ has

$$
K V(\sigma)=S_{K^{*}}^{ \pm}(\alpha) / J_{\sigma} \text { and } K V\left(p_{\tau, \sigma}\right)=q_{\tau, \sigma},
$$

where $J_{\sigma}$ denotes the ideal generated by the Euler classes $1-\alpha^{w_{c}}$ arising from the $w_{c}$ of (4.1) for $1 \leq c \leq n-d$. In this case, $P_{K}(\alpha ; \Sigma)$ is the $S_{K^{*}}^{ \pm}(\alpha)$-algebra of piecewise Laurent polynomials on $\Sigma$.

For $E=M U$, we work with graded commutative algebras over $M U_{T^{n}}^{*}$, whose structure is unknown. We therefore rely on the fact that every element of $M U_{T^{n}}^{*}$ is an even-dimensional linear combination of generators over $L^{*}$, and refer to $M U_{T^{n}}^{*}$ as the ring of $T^{n}$-cobordism forms. Such forms may not be representable by genuine $T^{n}$-manifolds, as exemplified by the Euler class $e\left(\alpha^{J}\right)$, whose homological dimension is -2 . This phenomenon arises from the lack of equivariant transversality, and the consequent failure of the Pontryagin-Thom construction to be epimorphic.

Example 4.14. The cobordism form diagram $M U V: \mathrm{CAT}^{o p}(\Sigma) \rightarrow \mathrm{GCALG}_{M U}$ has

$$
M U V(\sigma)=M U_{T^{n}}^{*} / J_{\sigma} \text { and } M U V\left(p_{\tau, \sigma}\right)=q_{\tau, \sigma},
$$


where $J_{\sigma}$ denotes the ideal generated by the Euler classes $e\left(\alpha^{w_{c}}\right)$ for $1 \leq c \leq n-d$. In this case, $P_{M U}(\Sigma)$ is the $M U_{T^{n}}^{*}$-algebra of piecewise cobordism forms on $\Sigma$.

For $E=H$, we work with graded commutative algebras over the polynomial algebra $S_{\mathbb{Z}}(x)$ of (3.7).

Example 4.16. The polynomial diagram $H V: \mathrm{CAT}^{o p}(\Sigma) \rightarrow \mathrm{GCALG}_{H}$ has

$$
H V(\sigma)=S_{\mathbb{Z}}(x) / J_{\sigma} \quad \text { and } H V\left(p_{\tau, \sigma}\right)=q_{\tau, \sigma},
$$

where $J_{\sigma}$ denotes the ideal generated by the Euler classes $w_{c}^{t r} x$ of $(4.1)$ for $1 \leq c \leq n-d$. In this case, $P_{H}(x ; \Sigma)$ is the $S_{\mathbb{Z}}(x)$-algebra of piecewise polynomials on $\Sigma$.

In $\left[\right.$ BFR1], $P_{H}(x ; \Sigma)$ is referred to as $P P_{\mathbb{Z}}(x ; \Sigma)$.

In Section 6 we invest $H_{T^{n}}^{*}(-)$ with various commutative integer-graded rings of coefficients $R$, which are zero in odd dimensions. The standard example $\mathbb{Z}$ is concentrated in dimension 0 , but we also consider $K \mathbb{Q}^{*}:=\mathbb{Q}\left[z, z^{-1}\right]$, where $z$ has cohomological dimension -2 , and

$$
H \wedge M U_{*}=H \wedge M U^{-*}:=H_{*}(M U) \cong S_{\mathbb{Z}}\left(b_{j}: j \geq 1\right),
$$

where $b_{j}$ has cohomological dimension $-2 j$ for every $j$. The corresponding spectrum is denoted by $E=H R$, and the analogue of diagram (4.13) by $H R V$; we continue to abbreviate $H \mathbb{Z}$ to $H$ in the standard example.

The equivariant coefficient ring $H R_{T^{n}}^{*}=H^{*}\left(B T^{n} ; R\right)$ must be identified with the completed tensor product $H^{*}\left(B T^{n}\right) \widehat{\otimes} R$. When $R=\mathbb{Z}$, the outcome is $H^{*}\left(B T^{n}\right)$; but for $K \mathbb{Q}^{*}$ or $H \wedge M U^{*}$, the ring

$$
H^{*}\left(B T^{n}\right) \widehat{\otimes} R \cong R[[x]]
$$

is an algebra of formal power series. It follows that $H R V(\sigma) \cong R[[x]] / J_{\sigma}$, and that $P_{H R}(x ; \Sigma)$ is the $R[[x]]$-algebra of piecewise formal power series on $\Sigma$.

We require two further $\Sigma^{o p}$-diagrams, obtained by applying Definition (4.6) to the Borel equivariant cohomology theories $E^{*}\left(E T^{n} \times_{T^{n}}-\right)$. In these cases the coefficients $E^{*}\left(B T^{n}\right)$ are also rings of formal power series.

The first such example identifies $K^{*}\left(B T^{n}\right)$ with $K^{*}[[\gamma]]$, on 0 -dimensional indeterminates $\gamma_{j}$ for $1 \leq j \leq n$.

Example 4.18. The Borel $K$-theory diagram $K_{B} V: \mathrm{CAT}^{o p}(\Sigma) \rightarrow \mathrm{GCALG}_{K_{B}}$ has

$$
K_{B} V(\sigma)=K^{*}[[\gamma]] / J_{\sigma},
$$

where $J_{\sigma}$ denotes the ideal generated by the Euler classes $(1+\gamma)^{w_{c}}-1$ for $1 \leq c \leq n-d$ and $K_{B} V\left(p_{\tau, \sigma}\right)$ is the natural projection. The limit $P_{K_{B}}(\gamma ; \Sigma)$ is the $K^{*}[[\gamma]]$-algebra of piecewise formal power series on $\Sigma$.

The second example identifies $M U^{*}\left(B T^{n}\right)$ with $L^{*}[[u]]$, on indeterminates $u_{j}$ of cohomological dimension 2 for $1 \leq j \leq n$.

Example 4.20. The Borel cobordism diagram $M_{B} V: \mathrm{CAT}^{o p}(\Sigma) \rightarrow \mathrm{GCALG}_{M U_{B}}$ has

$$
M U_{B} V(\sigma)=L^{*}[[u]] / J_{\sigma},
$$

where $J_{\sigma}$ denotes the ideal generated by the Euler classes $\left[w_{c, 1}\right]\left(u_{1}\right)+{ }_{U} \cdots+{ }_{U}\left[w_{c, n}\right]\left(u_{n}\right)$ (expressed in terms of the universal formal group law $U$ [Haz] over $\left.L^{*}\right)$, and $M U_{B} V\left(p_{\tau, \sigma}\right)$ is the natural projection. The limit $P_{M U_{B}}(u ; \Sigma)$ is the $L^{*}[[u]]$-algebra of piecewise formal power series on $\Sigma$.

In fact $P_{M U}(\Sigma)$ and $P_{M U_{B}}(u ; \Sigma)$ are the universal piecewise coefficient and piecewise formal power series algebras on $\Sigma$ respectively, for complex-oriented $E_{T^{n}}^{*}(-)$ and $E^{*}\left(E T^{n} \times_{T^{n}}-\right)$. The cases $P_{K}(\alpha ; \Sigma)$ and $P_{K_{B}}(\gamma ; \Sigma)$ correspond to the multiplicative formal group law, classified by the equivariant Todd genus. Similarly, $P_{H R}(x ; \Sigma)$ corresponds to the additive formal group law, classified by the Thom genus. 
Remark 4.22. A map of fans $\xi: \Sigma^{\prime} \rightarrow \Sigma$ may be interpreted as an $n \times n^{\prime}$ integer matrix $\xi$, for which the image $\xi\left(\sigma^{\prime}\right)$ of any cone $\sigma^{\prime}$ is contained in some cone $\sigma$. Let $\xi^{\dagger}\left(\sigma^{\prime}\right)$ be the minimal such $\sigma$. In each of the above cases, $\xi$ induces a natural transformation $\left(\xi^{\dagger}, \xi^{*}\right)$ of diagrams, and therefore a homomorphism $\xi^{*}$ of limits.

For example, in the case of $H V$, the homomorphism $\xi^{*}: H V(\sigma) \rightarrow H V\left(\sigma^{\prime}\right)$ is given in terms of the coordinate functions $x$ and $x^{\prime}$ by the matrix $\xi^{t r}$; it is well-defined because $w \in \xi^{\dagger}\left(\sigma^{\prime}\right)^{\perp}$ implies that $\xi^{t r} w \in\left(\sigma^{\prime}\right)^{\perp}$. In the case of $K V$, the homomorphism $\xi^{*}: K V(\sigma) \rightarrow K V\left(\sigma^{\prime}\right)$ is induced by $\xi^{*}\left(\alpha^{J}\right)=\left(\alpha^{\prime}\right)^{\xi^{t r} J}$, and is well-defined for similar reasons.

The construction of each piecewise algebra is therefore functorial (although care is required to check that $\dagger$ preserves composition). In particular, isomorphic fans yield isomorphic algebras.

Piecewise algebraic structures are natural generalizations of their global counterparts, and provide simple qualitative descriptions of algebras that may well be difficult to express in quantitative terms. For example, see [BFR1, Section 4], where $P_{H}\left(x ; \Sigma_{(1,2,3,4)}\right)$ is computed in terms of generators and relations. The simplest non-trivial divisive example is the following.

Example 4.23. The fan $\Sigma_{(1,1,2)}$ in $\mathbb{R}^{2}$ has seven cones: $\{0\}$; the three rays through $r_{0}=(-1,-2)$, $r_{1}=(1,0)$, and $r_{2}=(0,1)$; and three 2 -dimensional cones generated by all pairs of rays.

So $P_{H}(x)$ is an $S_{\mathbb{Z}}(x)$-algebra, where $x=\left(x_{1}, x_{2}\right)$. Calculations confirm that $P_{H}(x)$ is generated as a ring by four piecewise polynomials, namely the global linear functions $x_{1}$ and $x_{2}$, together with

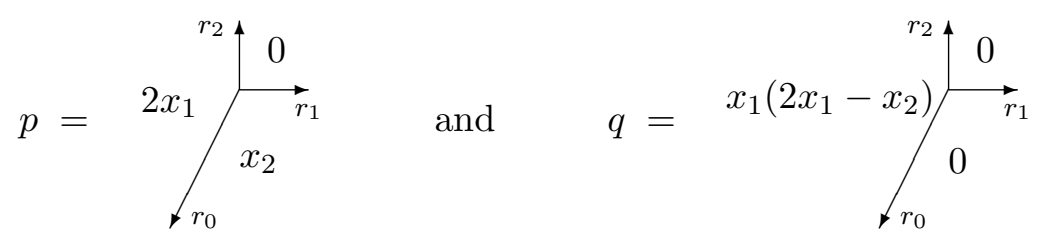

of degree 2 and 4 respectively. In fact $P_{H}(x)$ is isomorphic to $\mathbb{Z}\left[x_{1}, x_{2}, p, q\right] / I_{1}$, where $I_{1}$ is the ideal

$$
\left(p\left(p-x_{2}\right)-2 q, q\left(p-2 x_{1}\right), q\left(q-x_{1}\left(2 x_{1}-x_{2}\right)\right)\right) .
$$

As an $S_{\mathbb{Z}}(x)$-module, $P_{H}(x)$ has basis $\{1, p, q\}$.

Similarly, $P_{K}(\alpha)$ is an $S_{\mathbb{Z}}^{ \pm}(\alpha)$-algebra, where $\alpha=\left(\alpha_{1}, \alpha_{2}\right)$. Calculations confirm that $P_{K}(\alpha)$ is generated as a ring by six piecewise Laurent polynomials, namely the global elements $\alpha_{1}^{ \pm}$and $\alpha_{2}^{ \pm}$, together with

$$
\epsilon=1-\alpha_{1}^{2} \overbrace{1-\alpha_{2}}^{r_{2} \uparrow} r_{1} \quad \text { and } \quad \zeta=\left(1-\alpha_{1}\right)\left(\alpha_{2}-\alpha_{1}^{2}\right) \overbrace{r_{0}}^{r_{2}} \stackrel{r}{r}_{1}
$$

of grading (and virtual dimension) 0 . In fact $P_{K}(\alpha)$ is isomorphic to $S_{\mathbb{Z}}^{ \pm}\left(\alpha_{1}, \alpha_{2}\right)[\epsilon, \zeta] / I_{2}$, where $I_{2}$ is the ideal

$$
\left(\epsilon\left(\epsilon+\alpha_{2}-1\right)-\left(1+\alpha_{1}\right) \zeta, \zeta\left(\epsilon+\alpha_{1}^{2}-1\right), \zeta\left(\zeta-\left(1-\alpha_{1}\right)\left(\alpha_{2}-\alpha_{1}^{2}\right)\right)\right) .
$$

As an $S_{\mathbb{Z}}^{ \pm}(\alpha)$-module, $P_{K}(\alpha)$ has basis $\{1, \epsilon, \zeta\}$. An equivalent calculation of Anderson and Payne [AnPa, Example 1.6] interprets the latter in terms of an $R\left(T^{2}\right)$-module basis for the algebra of piecewise exponential functions on $\Sigma_{(1,1,2)}$.

\section{Cohomological applications}

In this section we prove Theorem 5.5 by translating the GKM-theoretic content of Proposition 3.10 into the piecewise algebraic language of Section 4. 
Our motivation lies in the results of [BFR1], which state that the Borel equivariant cohomology ring $H_{T^{n}}^{*}\left(X_{\Sigma} ; R\right)$ is isomorphic to $P_{H R}(x ; \Sigma)$ for any projective toric variety (smooth or singular) whose integral cohomology is concentrated in even dimensions. This may be thought of as a statement of compatibility with limits, in the sense that equivariant cohomology maps the homotopy colimit hocolim $V \simeq X_{\Sigma}$ to the algebraic $\operatorname{limit} \lim H R V=P_{H R}(x ; \Sigma)$. It follows from [BFR1] that the sequence

$$
0 \longrightarrow\left(S_{R}(x)\right) \longrightarrow P_{H R}(x ; \Sigma) \longrightarrow H^{*}\left(X_{\Sigma} ; R\right) \longrightarrow 0
$$

is short exact for any such variety. Furthermore, $P_{H R}(x ; \Sigma)$ is isomorphic to the face ring (or Stanley-Reisner algebra) $R[x ; \Sigma]$ for any smooth fan.

Working over a field immediately simplifies the situation; for example,

$$
H_{T^{n}}^{*}\left(X_{\Sigma} ; \mathbb{Q}\right) \cong P_{H \mathbb{Q}}(x ; \Sigma) \cong \mathbb{Q}[x ; \Sigma]
$$

holds for any fan $\Sigma$.

Kawasaki's calculations [Kaw] confirm that (5.1) is short exact for every weighted projective space. In other words, $P_{H R}\left(x ; \Sigma_{\chi}\right)$ reduces to the face ring whenever $\chi_{j}=1$ for every $0 \leq j \leq n$.

We now explain how to interpret the GKM description of Proposition 3.10 as the limit of an appropriate contravariant $\Sigma_{\chi}$ diagram. To proceed, we must therefore identify $\Sigma_{\chi}$ more explicitly. For general weights $\chi$, this may be difficult; in the divisive case, however, it is easy to specify the rays $r_{0}, \ldots, r_{n}$ precisely. Bearing in mind that $\chi$ is normalised (2.1), we set

$$
\left(\begin{array}{lll}
r_{0} & \ldots & r_{n}
\end{array}\right)=\left(\begin{array}{ccccc}
-1 & 1 & 0 & \ldots & 0 \\
-\chi_{2} & 0 & 1 & \ldots & 0 \\
\vdots & \vdots & \vdots & \ddots & \vdots \\
-\chi_{n} & 0 & 0 & \ldots & 1
\end{array}\right)
$$

as an $n \times(n+1)$ matrix. The cones $\sigma_{A}$ of $\Sigma_{\chi}$ are generated by rays $\left\{r_{i}: i \notin A\right\}$, as $A$ ranges over all non-empty strictly increasing subsequences $a_{1}, \ldots, a_{d}$ of $0, \ldots, n$. In particular, the $n$-dimensional cones are $\sigma_{0}, \ldots, \sigma_{n}$, and $\sigma_{A} \cap \sigma_{A^{\prime}}=\sigma_{A \cdot A^{\prime}}$ holds for any $A$ and $A^{\prime}$, where $A \cdot A^{\prime}$ is given by juxtaposition and reordering.

In order to study the diagrams $K V, M U V$ and $H V$ of Section 4, we must first identify the linear forms of (4.1) for $\Sigma_{\chi}$.

For every $0 \leq k<l \leq n$, the $(n-1)$-dimensional cone $\sigma_{k, l}$ is generated by the columns of the $n \times(n-1)$-matrix obtained from (5.3) by deleting columns $k$ and $l$. So a basis for $\mathbb{R}_{\sigma \frac{\perp}{,},}$ consists of a single primitive integral vector $w$, orthogonal to all remaining columns. If $1 \leq k$, then

$$
w=\left(0, \ldots, 0,-\chi_{l} / \chi_{k}, 0, \ldots, 0,1,0, \ldots, 0\right)
$$

(non-zero in positions $k$ and $l$ ) satisfies the conditions; if $k=0$, then $w=(0, \ldots, 0,1,0 \ldots, 0)$ suffices.

With reference to Examples 4.12, 4.14, and 4.16, we may now deduce the following.

Lemma 5.4. For any cone $\sigma_{k, l}$ and any $0 \leq k<l \leq n$ in $\Sigma_{\chi}$, the principal ideals $J_{k, l}:=J_{\sigma_{k, l}}$ in $S_{\mathbb{Z}}^{ \pm}(\alpha), M U_{T^{n}}^{*}$, and $S_{\mathbb{Z}}(x)$ are generated by $1-\alpha_{l} \alpha_{k}^{-\chi_{l} / \chi_{k}}, e\left(\alpha_{l} \alpha_{k}^{-\chi_{l} / \chi_{k}}\right)$, and $x_{l}-\left(\chi_{l} / \chi_{k}\right) x_{k}$ respectively, where $x_{0}=0$ and $\alpha_{0}=1$.

Lemma 5.4 summarizes the input required to prove Theorem 5.5.

Theorem 5.5. For any divisive weighted projective space $\mathbb{P}(\chi)$ :

1. $K_{T^{n}}^{*}(\mathbb{P}(\chi))$ is isomorphic as an $S_{K^{*}}^{ \pm}(\alpha)$-algebra to $P_{K}\left(\alpha ; \Sigma_{\chi}\right)$;

2. $M U_{T^{n}}^{*}(\mathbb{P}(\chi))$ is isomorphic as an $M U_{T^{n}}^{*}$-algebra to $P_{M U}\left(\Sigma_{\chi}\right)$;

3. $H_{T^{n}}^{*}(\mathbb{P}(\chi) ; R)$ is isomorphic as an $S_{R}(x)$-algebra to $P_{H R}\left(x ; \Sigma_{\chi}\right)$. 
Proof. We give the details for 1 .

Invoking (3.8) and Proposition 3.10, we must identify the algebra $\Gamma_{\mathbb{P}(\chi)}$ with $\lim _{\mathrm{GCALG}} K V$. The former is given by

$$
\left\{g=g(\alpha): 1-\alpha_{n-j} \alpha_{n-i}^{-\chi_{n-j} / \chi_{n-i}} \text { divides } g_{i}-g_{j} \text { for all } 0 \leq j<i \leq n\right\} \leq \prod_{i} S_{K^{*}}^{ \pm}(\alpha),
$$

and the universal properties of the latter suggest that we proceed by finding compatible homomorphisms $h_{a_{0}, \ldots, a_{d}}: \Gamma_{\mathbb{P}(\chi)} \rightarrow K V\left(\sigma_{a_{0}, \ldots, a_{d}}\right)$ for every cone $\sigma_{a_{0}, \ldots, a_{d}}$ in $\Sigma_{\chi}$. It follows from Example 4.12 and Lemma 5.4 that

$$
K V\left(\sigma_{a_{0}, \ldots, a_{d}}\right)=S_{K^{*}}^{ \pm}(\alpha) / J_{a_{0}, \ldots, a_{d}},
$$

where $J_{a_{0}, \ldots, a_{d}}$ denotes the ideal generated by the Laurent polynomials $1-\alpha_{l} \alpha_{k}^{-\chi_{l} / \chi_{k}}$ as $k, l$ ranges over the length 2 subsequences of $0 \leq a_{0}, \ldots, a_{n} \leq n$.

Given any $g$ in $\Gamma_{\mathbb{P}(\chi)}$, we first consider cones of dimension $n$, and define $h_{k}(g):=g_{n-k}$ in $S_{K^{*}}^{ \pm}(\alpha)$ for every $0 \leq k \leq n$. On cones of dimension $n-1$, we let

$$
h_{k, l}(g):=g_{n-k} \equiv g_{n-l} \bmod 1-\alpha_{l} \alpha_{k}^{-\chi_{l} / \chi_{k}}
$$

in $K V\left(\sigma_{k, l}\right)=S_{K^{*}}^{ \pm}(\alpha) /\left(1-\alpha_{l} \alpha_{k}^{-\chi_{l} / \chi_{k}}\right)$, for every $0 \leq k<l \leq n$. This is well-defined, because $1-\alpha_{l} \alpha_{k}^{-\chi_{l} / \chi_{k}}$ divides $g_{n-k}-g_{n-l}$ in $S_{K^{*}}^{ \pm}(\alpha)$. The definition extends to

$$
h_{a_{0}, \ldots, a_{d}}\left(g_{i}\right):=g_{n-a_{0}} \equiv \cdots \equiv g_{n-a_{d}} \bmod J_{a_{0}, \ldots, a_{d}}
$$

for any $2 \leq d \leq n$, because the $g_{n-a_{0}}, \ldots, g_{n-a_{d}}$ satisfy precisely the required pairwise divisibility conditions in $S_{K^{*}}^{ \pm}(\alpha)$. Moreover, $h_{a_{0}, \ldots, a_{d}}$ is a homomorphism of $S_{K^{*}}^{ \pm}(\alpha)$-algebras, by definition.

In order to confirm the compatibility of the $h_{A}$ over $\mathrm{CAT}^{o p}\left(\Sigma_{\chi}\right)$, we note that every morphism takes the form $\sigma_{A} \supseteq \sigma_{A \cdot A^{\prime}}$. The corresponding projection $q_{\sigma_{A}, \sigma_{A \cdot A^{\prime}}}: K V\left(\sigma_{A}\right) \rightarrow K V\left(\sigma_{A \cdot A^{\prime}}\right)$ is induced by the inclusion $J_{A} \leq J_{A \cdot A^{\prime}}$, which adjoins the expressions $1-\alpha_{l} \alpha_{k}^{-\chi_{l} / \chi_{k}}$ as $k, l$ ranges over the length 2 subsequences of $A^{\prime}$ : so compatibility is assured. We have therefore constructed a homomorphism $h: \Gamma_{\mathbb{P}(\chi)} \rightarrow \lim _{\mathrm{GCALG}} K V$ of $S_{K^{*}}^{ \pm}(\alpha)$-algebras.

We conclude by showing that $h$ is automatically an isomorphism. Given distinct elements $g$ and $g^{\prime}$ of $\Gamma_{\mathbb{P}(\chi)}$, there must exist at least one $k$ such that $g_{k} \neq g_{k}^{\prime}$ as elements of $S_{K^{*}}^{ \pm}(\alpha)$; hence $h_{k}(g) \neq h_{k}\left(g^{\prime}\right)$ in $K V\left(\sigma_{k}\right)$, and $h$ is monic. Similarly, any element $\left(g_{A}\right)$ of $\lim _{\mathrm{GCALG}} K V$ determines $\left(g_{a}\right)$ in $\Gamma_{\mathbb{P}(\chi)}$, by restricting to $n$-dimensional cones; thus $h\left(g_{a}\right)=\left(g_{A}\right)$, and $h$ is epic.

The entire argument applies to $\mathbf{2}$ and $\mathbf{3}$, with minor modifications. For $H_{T^{n}}^{*}(\mathbb{P}(\chi) ; R)$, the statement is also a special case of [BFR1, Proposition 2.2].

Informally, the connection between $T^{n}$-equivariant bundles over $X_{\Sigma}$ and piecewise Laurent polynomials on $\Sigma$ is easy to make. Every such bundle determines a representation of $T^{n}$ on the fibre at each fixed point, and therefore on each maximal cone. These representations must be compatible over any $T^{n-1}$-invariant $S^{2}$ containing two fixed points, and therefore on cones of codimension 1.

The close relationship between GKM theory and piecewise algebra has long been known over fields of characteristic zero, and Theorem 5.5 is an instance of its extension to integral situations.

\section{Completion and Borel cohomology}

In this section we introduce the completions of $K_{T^{n}}^{*}(\mathbb{P}(\chi))$ and $M U_{T^{n}}^{*}(\mathbb{P}(\chi))$ at their augmentation ideals, and discuss their relationships with the Borel equivariant cohomology ring of $\mathbb{P}(\chi)$ under the Chern character and the Boardman homomorphism respectively. We express our results in terms of certain natural transformations between diagrams of Section 4.

Definitions 6.1. The $K$-theory completion transformation $\wedge:=\wedge^{K}: K V \rightarrow K_{B} V$ is defined on objects by the ring homomorphisms

$$
S_{K^{*}}^{ \pm}(\alpha) / J_{\sigma} \longrightarrow K^{*}[[\gamma]] / J_{\sigma},
$$


where $\wedge\left(\alpha_{j}\right)=1-\gamma_{j}$ and $\wedge\left(\alpha_{j}^{-1}\right)=1+\sum_{i \geq 1} \gamma_{j}^{i}$ for $1 \leq j \leq n$; on morphisms, $\wedge$ maps the first natural projection to the second.

The cobordism completion transformation $\wedge:=\wedge^{M U}: M U V \rightarrow M U_{B} V$ is defined on objects by the ring homomorphisms

$$
M U_{T^{n}}^{*} / J_{\sigma} \longrightarrow L^{*}[[u]] / J_{\sigma},
$$

where $\wedge\left(e_{T^{n}}\left(\alpha_{j}\right)\right)=u_{j}$ and $\wedge\left(e_{T^{n}}\left(\alpha_{j}^{-1}\right)\right)=[-1]\left(u_{j}\right)$ for $1 \leq j \leq n$; on morphisms, $\wedge$ maps the first natural projection to the second.

So $\wedge^{K}$ is induced by the homomorphism

$$
S_{K^{*}}^{ \pm}(\alpha) \longrightarrow K^{*}[[\gamma]]
$$

representing completion at the augmentation ideal $I$ [AM, Chapter 10]. It is well-defined because $\wedge\left(\alpha_{j}^{-1}\right)=\left(\wedge\left(\alpha_{j}\right)\right)^{-1}$ for $1 \leq j \leq n$ and $\wedge\left(\alpha^{w_{c}}\right)=(1-\gamma)^{w_{c}}$ for $1 \leq c \leq n-d$, so that $\wedge$ maps $J_{\sigma}$ to $J_{\sigma}$. The augmentation $S_{K^{*}}^{ \pm}(\alpha) \rightarrow K^{*}$ assigns to each virtual representation its dimension.

Similarly, $\wedge^{M U}$ is induced by the homomorphism

$$
M U_{T^{n}}^{*} \longrightarrow L^{*}[[u]]
$$

representing completion at the augmentation ideal $I$. It is well-defined because $\wedge\left(\alpha^{w_{c}}\right)=\left[w_{c, 1}\right]\left(u_{1}\right)+U$ $\cdots+{ }_{U}\left[w_{c, n}\right]\left(u_{n}\right)$ for $1 \leq c \leq n-d$, so that $\wedge$ maps $J_{\sigma}$ to $J_{\sigma}$. The augmentation $M U_{T^{n}}^{*} \rightarrow L^{*}$ forgets the $T^{n}$-action on each equivariant cobordism class.

Definitions 6.4. The Chern transformation $c t: K_{B} V \rightarrow H\left(K \mathbb{Q}^{*}\right) V$ is given on objects by the homomorphisms

$$
K^{*}[[\gamma]] / J_{\sigma} \longrightarrow S_{K \mathbb{Q}^{*}}(x) / J_{\sigma},
$$

where $c t\left(\gamma_{j}\right)=1-e^{z x_{j}}$ for $1 \leq j \leq n$, and $c t$ embeds the scalars $K^{*}$ as $K^{*} \otimes 1$ in $K \mathbb{Q}^{*}:=K^{*} \otimes \mathbb{Q}$; on morphisms, ct maps the first natural projection to the second.

The Boardman transformation bt: $M U_{B} V \rightarrow H\left(H \wedge M U^{*}\right) V$ is given on objects by the homomorphisms

$$
L^{*}[[u]] / J_{\sigma} \longrightarrow S_{H \wedge M U^{*}}(x) / J_{\sigma},
$$

where $b t\left(u_{j}\right)=\sum_{i \geq 0} b_{i} x_{j}^{i+1}$ for $1 \leq j \leq n$, and $b t$ embeds the scalars $L^{*}$ in $H \wedge M U^{*}$ via the Hurewicz homomorphism; on morphisms, bt maps the first natural projection to the second.

So $c t$ and $b t$ are induced by the respective homomorphisms

$$
K^{*}[[\gamma]] \longrightarrow S_{K \mathbb{Q}^{*}}(x) \text { and } L^{*}[[u]] \longrightarrow S_{H \wedge M U^{*}}(x),
$$

and are well-defined because $J_{\sigma}$ maps to $J_{\sigma}$ in each instance.

The commutativity of the diagrams required for the naturality of $\wedge, c t$, and $b t$ follow directly from the definitions. They therefore induce morphisms of limits, and so define homomorphisms

$$
\wedge^{K}: P_{K}(\alpha ; \Sigma) \longrightarrow P_{K_{B}}(\gamma ; \Sigma) \text { and } c t: P_{K_{B}}(\gamma ; \Sigma) \longrightarrow P_{H\left(K \mathbb{Q}^{*}\right)}(x ; \Sigma)
$$

and

$$
\wedge^{M U}: P_{M U}(\Sigma) \longrightarrow P_{M U_{B}}(u ; \Sigma) \quad \text { and } \quad b t: P_{M U_{B}}(u ; \Sigma) \longrightarrow P_{H\left(H \wedge M U^{*}\right)}(x ; \Sigma)
$$

of piecewise structures. In particular, $\wedge^{K}$ and $\wedge^{M U}$ may be viewed as conewise completions; but completion commutes with limits, so they coincide with the respective completions of $P_{K}(\alpha ; \Sigma)$ and $P_{M U}(\Sigma)$ at their augmentation ideals $I$. Similarly, ct and bt are the conewise Chern and conewise Boardman homomorphism respectively.

Note that $\wedge^{K}$ and $\wedge^{M U}$ are morphisms of algebras over the respective completion homomorphisms (6.2) and (6.3) of scalars. Furthermore, ct and bt arise from conewise rational isomorphisms, and are therefore rational isomorphisms themselves; they are also morphisms of algebras over the homomorphisms (6.5). In other words, (6.6) and (6.7) describe the extensions of (6.2), (6.3), and (6.5) to the piecewise setting. 
Remarks 6.8. The composition $c t \circ \wedge^{K}$ is a natural transformation $c c: K V \rightarrow H\left(K \mathbb{Q}^{*}\right) V$. It is induced by the homomorphism

$$
c c: S_{K^{*}}^{ \pm}(\alpha) \longrightarrow S_{K \mathbb{Q}^{*}}(x),
$$

which satisfies $c c\left(\alpha_{j}\right)=e^{z x_{j}}$ for all $1 \leq j \leq n$. On limits,

$$
c c: P_{K}(\alpha ; \Sigma) \longrightarrow P_{H\left(K \mathbb{Q}^{*}\right)}(x ; \Sigma)
$$

identifies $P_{K}(\alpha ; \Sigma)$ with a subring of piecewise formal exponential functions (the viewpoint adopted by $[\mathrm{AnPa}]$, and anticipated in Example 4.23). It is a morphism of algebras over the homomorphism (6.9) of scalars.

Similarly, $b t \circ \wedge^{M U}$ is a natural transformation $b c: M U V \rightarrow H\left(H \wedge M U^{*}\right) V$. It is induced by the homomorphism

$$
\text { bc: } M U_{T^{n}}^{*} \longrightarrow S_{H \wedge M U^{*}}(x),
$$

which satisfies $b c\left(e\left(\alpha_{j}\right)\right)=\sum_{i \geq 0} b_{i} x_{j}^{i+1}$ for all $1 \leq j \leq n$. On limits,

$$
b c: P_{M U}(\Sigma) \longrightarrow P_{H\left(H \wedge M U^{*}\right)}(x ; \Sigma)
$$

identifies $P_{M U}(\Sigma)$ with a subring of piecewise formal power series; it is a morphism of algebras over the homomorphism (6.10) of scalars.

Using the isomorphism of Theorem 5.5.2, we may now interpret the homomorphisms (6.6) and (6.7) topologically. We deal first with $K$-theory.

For any $T^{n} \odot Y$, the Borel equivariant $K$-theory $K^{*}\left(E T^{n} \times_{T^{n}} Y\right)$ is an algebra over the coefficient ring $K^{*}\left(B T^{n}\right)$, which acts via the projection map $\pi$ of the $T^{n}$-bundle

$$
Y \longrightarrow E T^{n} \times_{T^{n}} Y \stackrel{\pi}{\longrightarrow} B T^{n} .
$$

Atiyah and Segal define a homomorphism $\lambda: K_{T^{n}}^{*}(Y) \rightarrow K^{*}\left(E T^{n} \times_{T^{n}} Y\right)$ by assigning the vector bundle $\left(E T^{n} \times \theta\right) / T^{n}$ to each $T^{n}$-equivariant vector bundle $\theta$ over $Y$. For compact spaces such as $X_{\Sigma}$, they prove [AS1, Theorem 2.1] that $\lambda$ is completion at the augmentation ideal $I$. If, for example, $Y$ is the 1-point $T^{n}$-space $*$, then $\lambda: K_{T^{n}}^{*} \rightarrow K^{*}\left(B T^{n}\right)$ corresponds to the completion map (6.2), and identifies the coefficients $K^{*}\left(B T^{n}\right)$ with $K^{*}[[\gamma]]$, as in Example 4.18. Furthermore, $\gamma_{j}$ is the $K$-theoretic Euler class of the $j$ th canonical line bundle over $B T^{n}$, for $1 \leq j \leq n$. In general, $\lambda$ may be interpreted as converting the $S_{K^{*}}^{ \pm}(\alpha)$-algebra structure of $K_{T^{n}}^{*}(Y)$ to the $K^{*}[[\gamma]]$-algebra structure of $K^{*}\left(E T^{n} \times_{T^{n}} Y\right)$.

The Chern character ch: $K^{*}\left(E T^{n} \times_{T^{n}} Y\right) \rightarrow H^{*}\left(E T^{n} \times_{T^{n}} Y ; K \mathbb{Q}^{*}\right)=: H_{T^{n}}^{*}\left(Y ; K \mathbb{Q}^{*}\right)$ is the natural transformation of cohomology theories induced by the Hurewicz morphism

$$
K \simeq S^{0} \wedge K \stackrel{i \wedge 1}{\longrightarrow} H \wedge K
$$

of complex-oriented ring spectra, where $i$ denotes the unit of the integral Eilenberg-Mac Lane spectrum $H$. On coefficient rings, it embeds $K^{*} \cong \mathbb{Z}\left[z, z^{-1}\right]$ in $H \wedge K^{*} \cong \mathbb{Q}\left[z, z^{-1}\right]=K \mathbb{Q}^{*}$ by $\mathbb{Z}<\mathbb{Q}$, and on $\mathbb{C} P^{\infty}$ it embeds $K^{*}\left(\mathbb{C} P^{\infty}\right) \cong K^{*}\left[\left[\gamma_{1}\right]\right]$ in $H^{*}\left(\mathbb{C} P^{\infty} ; K \mathbb{Q}^{*}\right) \cong S_{K \mathbb{Q}^{*}}\left(x_{1}\right)$ by $\operatorname{ch}\left(\gamma_{1}\right)=1-e^{z x_{1}}$. Further properties of ch may be found in [Hi, Chapter 5], for example; in particular, it is always a rational isomorphism.

Theorem 6.13. For any divisive weighted projective space, $K^{*}\left(E T^{n} \times_{T^{n}} \mathbb{P}(\chi)\right)$ is isomorphic as a $K^{*}[[\gamma]]$-algebra to $P_{K_{B}}\left(\gamma ; \Sigma_{\chi}\right)$; with respect to this identification, the Atiyah-Segal completion map $\lambda: K_{T^{n}}^{*}(\mathbb{P}(\chi)) \rightarrow K^{*}\left(E T^{n} \times_{T^{n}} \mathbb{P}(\chi)\right)$ corresponds to the conewise completion homomorphism $\wedge^{K}$, and the Chern character ch: $K^{*}\left(E T^{n} \times_{T^{n}} \mathbb{P}(\chi)\right) \rightarrow H_{T^{n}}^{*}\left(\mathbb{P}(\chi) ; K \mathbb{Q}^{*}\right)$ corresponds to the conewise Chern transformation ct. 
Proof. Theorem 5.5.1 shows that $\lambda$ corresponds to $\wedge$, and has target $P_{K_{B}}\left(\gamma ; \Sigma_{\chi}\right)$; so the latter is necessarily isomorphic to $K^{*}\left(E T^{n} \times_{T^{n}} \mathbb{P}(\chi)\right)$ as a $K^{*}[[\gamma]]$-algebra.

Moreover, ch: $K^{*}\left(B T^{n}\right) \rightarrow H^{*}\left(B T^{n} ; K \mathbb{Q}^{*}\right)$ maps $\gamma_{j}$ to $1-e^{z x_{j}}$ for every $1 \leq j \leq n$, which agrees precisely with $c t$ as specified in (6.5). Since $c h$ is natural with respect to the inclusion $\amalg B T^{n} \subset E T^{n} \times_{T^{n}} \mathbb{P}(\chi)$ induced by the fixed point set, the result follows.

We may extend the isomorphism of Theorem 6.13 to any projective toric variety $X_{\Sigma}$ for which $H^{*}\left(X_{\Sigma}\right)$ is torsion free and concentrated in even dimensions. We interpret $P_{K_{B}}(\gamma ; \Sigma)$ as a $K^{*}[[\gamma]]$ subalgebra of $\prod K^{*}[[\gamma]]$, where the latter contains one factor for each maximal cone of $\Sigma$, and hence for each $T^{n}$-fixed point.

Theorem 6.14. The Borel equivariant $K$-theory of any such $X_{\Sigma}$ is isomorphic as a $K^{*}[[\gamma]]$-algebra to $P_{K_{B}}(\gamma ; \Sigma)$.

Proof. By [BFR1, Proposition 2.2], $H_{T^{n}}^{*}\left(X_{\Sigma}\right)$ is also torsion free and even dimensional, and isomorphic to $P_{H}(x ; \Sigma)$. So the Chern character $c h: K^{*}\left(E T^{n} \times_{T^{n}} X_{\Sigma}\right) \rightarrow H_{T^{n}}^{*}\left(X_{\Sigma} ; K \mathbb{Q}^{*}\right)$ is monic, and identifies $K^{*}\left(E T^{n} \times_{T^{n}} X_{\Sigma}\right)$ with a subring of $P_{H\left(K \mathbb{Q}^{*}\right)}(x ; \Sigma)$. By the naturality of ch, the inclusion of the fixed point set and the projection of (6.11) induce a commutative diagram

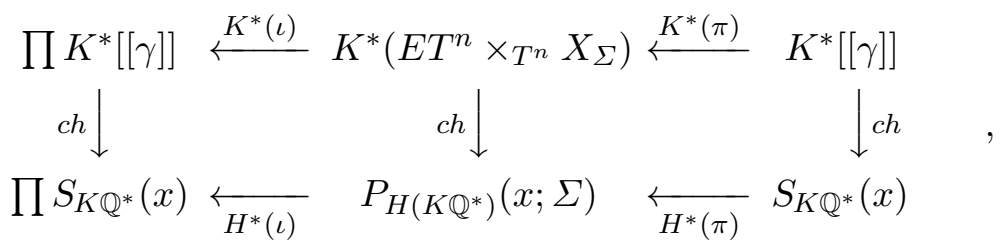

in which $H^{*}(\iota), H^{*}(\pi), K^{*}(\pi)$, and all maps $c h$, are monic. It follows that $K^{*}(\iota)$ is also monic, and that we may identify the elements $\gamma_{j}$ in $K^{*}\left(E T^{n} \times_{T^{n}} X_{\Sigma}\right)$, for $1 \leq j \leq n$. The image of $K^{*}(\iota)$ automatically lies in the subalgebra $P_{K_{B}}(\gamma ; \Sigma)<\prod K^{*}[[\gamma]]$, because $\iota$ factors through the equivariant 1-skeleton of $X_{\Sigma}$; so it remains to show that the image is the entire subalgebra.

Note that the augmentation ideal $I$ of $K^{*}[[\gamma]]$ is generated by the $\gamma_{j}$, and its image $\operatorname{ch}(I)=(x)$ is generated by the $x_{j}$. Furthermore, the filtration by powers of $I$ coincides with the skeletal filtration for $K^{*}\left(B T^{n}\right)$.

Choose $f:=f(\gamma)$ in $P_{K_{B}}(\gamma ; \Sigma)$, and assume that its augmentation is zero without loss of generality. So $\operatorname{ch}(f)=f\left(1-e^{z x}\right)$ is a piecewise formal power series in $\prod S_{K \mathbb{Q}^{*}}(x)$, and may be rewritten as a piecewise polynomial in the variables $x$ over $K \mathbb{Q}^{*}$. As such, it takes the form $H^{*}(\iota)\left(f^{\prime}\right)$ for some element $f^{\prime}=f^{\prime}(x)$ in $P_{H\left(K \mathbb{Q}^{*}\right)}(x ; \Sigma)$. Since $f^{\prime}(0)=0$, it has filtration $q_{1} \geq 1$ with respect to $(x)$; so the integrality properties of $c h$ ensure the existence of an element $f_{1}=f_{1}(x)$ in $K^{*}\left(E T^{n} \times_{T^{n}} X_{\Sigma}\right)$, such that $c h\left(f_{1}\right) \equiv f^{\prime} \bmod I^{q_{1}+1}$. Hence $K^{*}(\iota)\left(f_{1}\right) \equiv f \bmod I^{q_{1}+1}$ in $P_{K_{B}}(\gamma ; \Sigma)$.

Now iterate this procedure on $f-K^{*}(\iota)\left(f_{1}\right)$, to obtain a sequence of elements $\left(f_{n}=f_{n}(x)\right)$ in $K^{*}\left(E T^{n} \times_{T^{n}} X_{\Sigma}\right)$ for which

$$
K^{*}(\iota)\left(f_{n}\right) \equiv f-K^{*}(\iota)\left(f_{1}+\cdots+f_{n-1}\right) \bmod I^{q_{n}+1},
$$

with $q_{1}<\cdots<q_{n}$. Since $K^{*}\left(E T^{n} \times_{T^{n}} X_{\Sigma}\right)$ and $P_{K_{B}}(\gamma ; \Sigma)$ are $I$-adically complete, it follows that $f_{1}+\cdots+f_{n}+\cdots$ converges to an element $f^{\circ}$ in the former, and that $K^{*}(\iota)\left(f^{\circ}\right)=f$ in the latter. So $K^{*}(\iota)$ is epic, as required.

We now turn to the cobordism versions of our previous two results.

In [tD], tom Dieck introduces the bundling transformation $\alpha: M U_{T^{n}}^{*}(Y) \rightarrow M U^{*}\left(E T^{n} \times_{T^{n}} Y\right)$, which is proven in $[\mathrm{CM}]$ to be completion at the augmentation ideal. If $Y=*$, then $\alpha$ reduces to the homomorphism (6.3), and identifies $M U^{*}\left(B T^{n}\right)$ with $M U^{*}[[u]]$, as in Example 4.20. Each $u_{j}$ is the cobordism Euler class of the $j$ th canonical line bundle over $B T^{n}$, for $1 \leq j \leq n$. 
The Boardman homomorphism bh: $M U^{*}\left(E T^{n} \times_{T^{n}} Y\right) \rightarrow H_{T^{n}}^{*}\left(Y ; H \wedge M U^{*}\right)$ is induced by the Hurewicz morphism

$$
M U \simeq S^{0} \wedge M U \stackrel{i \wedge 1}{\longrightarrow} H \wedge M U
$$

by analogy with (6.12). On coefficient rings it embeds $L^{*}$ in $H \wedge M U^{*}$ by the Hurewicz homomorphism, and on $\mathbb{C} P^{\infty}$ it embeds $M U^{*}\left(\mathbb{C} P^{\infty}\right) \cong L^{*}\left[\left[u_{1}\right]\right]$ in $H \wedge M U^{*}\left(\mathbb{C} P^{\infty}\right) \cong H_{*}(M U)\left[\left[x_{1}\right]\right]$ by $b h\left(u_{1}\right)=\sum_{i \geq 0} b_{i} x_{1}^{i+1}$. Further properties of $b h$ may be found in $[\mathrm{A}]$, for example.

We are now in a position to state the cobordism versions of Theorems 6.13 and 6.14 ; they are verified by substituting $b h$ for $c h$ in each of the proofs.

Theorem 6.16. For any divisive weighted projective space, $M U^{*}\left(E T^{n} \times_{T^{n}} \mathbb{P}(\chi)\right)$ is isomorphic as an $L^{*}[[u]]$-algebra to $P_{M U_{B}}(u ; \Sigma)$; with respect to this identification, the bundling transformation $\alpha: M U_{T^{n}}^{*}(\mathbb{P}(\chi)) \rightarrow M U^{*}\left(E T^{n} \times_{T^{n}} \mathbb{P}(\chi)\right)$ corresponds to the conewise completion homomorphism $\wedge^{M U}$, and the Boardman homomorphism bh: $M^{*}\left(E T^{n} \times_{T^{n}} \mathbb{P}(\chi)\right) \rightarrow H_{T^{n}}^{*}\left(\mathbb{P}(\chi) ; H \wedge M U^{*}\right)$ corresponds to the conewise Boardman transformation bt.

Theorem 6.17 applies to projective toric varieties $X_{\Sigma}$ whose integral cohomology is free and even.

Theorem 6.17. The Borel equivariant $M U$-theory of any such $X_{\Sigma}$ is isomorphic as an $L^{*}[[u]]$ algebra to $P_{M U_{B}}(u ; \Sigma)$.

For the proof, we interpret $P_{M U_{B}}(u ; \Sigma)$ as an $L^{*}[[u]]$-subalgebra of $\prod L^{*}[[u]]$.

Note that Theorems 6.14 and 6.17 apply to all smooth toric varieties, and to a wider class of singular examples than Theorem 5.5; in particular, they hold for iterated products of arbitrary weighted projective spaces. They therefore provide evidence for the conjecture that $K_{T^{n}}^{*}\left(X_{\Sigma}\right)$ and $M U_{T^{n}}^{*}\left(X_{\Sigma}\right)$ are isomorphic to $P_{K}(\alpha ; \Sigma)$ and $P_{M U}(\Sigma)$ respectively, for any projective toric variety whose integral cohomology is free and even. Without further proof, however, the most we can claim is that each pair of algebras share the same completion.

Combining Theorem 6.17 with [KU, Theorem 6.4] confirms that the equivariant algebraic cobordism ring of a smooth projective toric variety $Y$ is isomorphic to $M U^{*}\left(E T^{n} \times_{T^{n}} Y\right)$. This fact also follows from [KK, Theorem 3.7]. Most recently, Gonzalez and Karu have defined operational equivariant algebraic cobordism, and [GoKa, Theorem 7.2] proves that their ring is isomorphic to $M U^{*}\left(E T^{n} \times_{T^{n}} Y\right)$ for any quasiprojective toric variety $Y$ to which Theorem 6.17 applies, singular or otherwise. No analogous coincidences arise in Sections 5 or 7 , because the coefficient rings $L^{*}[[u]]$ of the algebraic theories are complete.

\section{THE SMOOTH CASE}

A version of Theorem 5.5 for smooth fans may well be known to experts, but statements are difficult to find in the literature. There are, however, explicit references such as [VV] and [AnPa] to analogous results in equivariant algebraic and operational $K$-theory respectively. The goal of this section is to outline a proof (and certain consequences) of Theorem 5.5 for smooth polytopal fans $\Sigma$, thereby confirming that all three forms of $K$-theory agree on the corresponding $X_{\Sigma}$. In this context, polytopal indicates that $\Sigma$ is the normal fan of a compact simple polytope $P_{\Sigma}$, and therefore is complete. We expect that our results may be extended to more general fans by applying the methods of $[\mathrm{Fr}]$.

Our proof relies on the work of Harada and Landweber [HaL], which deals with symplectic manifolds $(M, \omega)$ equipped with a Hamiltonian action of $T^{n}$. In [HaL, Definition 4.1], such an action is defined to be GKM whenever the fixed point set is finite and the isotropy weights at each fixed point $p$ are pairwise linearly independent. The latter requirement may be restated in the notation of Section 3 by decomposing the $n$-dimensional representation $\rho_{p}$ of $T^{n}$ on $T_{p}(M)$ as a sum $\bigoplus_{j=1}^{n} \alpha^{J_{p, j}}$ of 1 -dimensionals, and insisting that the $J_{p, j}$ are pairwise linearly independent in $\mathbb{Z}^{n}$ for each fixed point $p$. 
Let $\Sigma$ be smooth and polytopal, so that $X_{\Sigma}$ is smooth, compact and projective. In fact, $X_{\Sigma}$ is also a symplectic toric manifold in the sense of [CdS, Part XI], with respect to the induced symplectic structure arising from its projective embedding. The associated moment map $\Phi$ can be identified with the orbit map $X_{\Sigma} \rightarrow X_{\Sigma} / T^{n} \cong P_{\Sigma}$; moreover, $P_{\Sigma}$ is a Delzant polytope [De, CdS]. The fixed points of the action are precisely the inverse images of the vertices of $P_{\Sigma}$; in particular, the set of fixed points is finite. Furthermore, since $P_{\Sigma}$ is Delzant, the edges incident on any vertex $t$ of $P_{\Sigma}$ are specified by $n$ primitive integral vectors $J_{t, s_{1}}, \ldots, J_{t, s_{n}}$, which form a basis for the standard lattice $\mathbb{Z}^{n}$ in the ambient $\mathbb{R}^{n}$. Then $T^{n} \subset X_{\Sigma}$ is given in a neighborhood of $t$ by the representation $\alpha_{t}:=\bigoplus_{j=1}^{n} \alpha^{J_{t, s_{j}}}$, and the action is GKM.

We may now apply [HaL, Theorem 4.4] to $T^{n} \odot X_{\Sigma}$, by noting that $\Phi$ has compact domain, so every component is proper and bounded below.

Theorem 7.1. For any smooth polytopal fan $\Sigma$, the inclusion of the fixed point set induces an isomorphism of $K_{T^{n}}^{*}\left(X_{\Sigma}\right)$ with

$$
\Gamma_{\Sigma}=\left\{y: 1-\alpha^{J_{t, s}} \text { divides } y_{s}-y_{t} \text { for all } s \prec t\right\} \leq \prod_{t} K_{T^{n}}^{*},
$$

where $t$ ranges over the vertices of $P_{\Sigma}$.

Theorem 7.1 also holds for $M U_{T^{n}}^{*}\left(X_{\Sigma}\right)$, following our observations on $M U_{T^{n}}$-Euler classes in Section 3.

Corollary 7.2. For any smooth polytopal fan $\Sigma$ :

1. $K_{T^{n}}^{*}\left(X_{\Sigma}\right)$ is isomorphic as an $S_{K^{*}}^{ \pm}(\alpha)$-algebra to $P_{K}(\alpha ; \Sigma)$;

2. $M U_{T^{n}}^{*}\left(X_{\Sigma}\right)$ is isomorphic as an $M U_{T^{n}}^{*}$-algebra to $P_{M U}(\Sigma)$;

3. $H_{T^{n}}^{*}\left(X_{\Sigma} ; R\right)$ is isomorphic as an $S_{R}(x)$-algebra to $P_{H R}(x ; \Sigma)$.

Corollary 7.2 follows from Theorem 7.1 by adapting the proof of Theorem 5.5.

Since $\Sigma$ is smooth, each algebra of Corollary 7.2 admits an alternative description in terms of the face ring $R[\Sigma]$, inspired by the isomorphism $H_{T^{n}}^{*}\left(X_{\Sigma} ; R\right) \cong R[\Sigma]$ mentioned in Section 5 . The face ring associates 2 -dimensional indeterminates $y_{j}$ to the the rays $v_{j}$ of $\Sigma$ for $1 \leq j \leq m$, and may first have been described as a limit in [PRV].

Given any set $\omega$ of rays, it is convenient to denote the set of variables $\left\{y_{j}: v_{j} \in \omega\right\}$ by $y(\omega)$, and to abbreviate the monomial $\prod_{y(\omega)} y_{j}$ to $y_{\omega}$.

Definition 7.3. The face diagram $S_{R}: \mathrm{CAT}^{o p}(\Sigma) \rightarrow$ GCALG has

$$
S_{R}(\sigma)=S_{R}(y(\sigma)) \text { and } S_{R}\left(p_{\tau, \sigma}\right)=f_{\tau, \sigma},
$$

where $f_{\tau, \sigma}: S_{R}(\tau) \rightarrow S_{R}(\sigma)$ is induced by annihilating those $y_{i}$ for which $v_{i} \in \tau \backslash \sigma$.

The face ring $R[\Sigma]=R[y ; \Sigma]$ is the limit of $S_{R}$, and is additively generated by those monomials $y^{J}:=y_{1}^{j_{1}} \cdots y_{m}^{j_{m}}$ whose support $\prod_{j_{k} \neq 0} y_{k}$ is $y_{\sigma}$ for some cone $\sigma$, where $J=\left(j_{1}, \ldots, j_{m}\right)$ lies in $\mathbb{Z}_{\geq}^{m}$. There is therefore a canonical isomorphism

$$
S_{R}(y) /\left(y_{\omega}: \omega \notin \Sigma\right) \stackrel{\cong}{\longrightarrow} R[y ; \Sigma]
$$

of graded $S_{R}(y)$-algebras.

The version required for $K_{T^{n}}^{*}\left(X_{\Sigma}\right)$ involves 0-dimensional indeterminates $\beta_{j}$, for $1 \leq j \leq m$.

Definition 7.5. The Laurent face diagram $S_{K^{*}}^{ \pm}$CAT ${ }^{o p}(\Sigma) \rightarrow$ GCALG has

$$
S_{K^{*}}^{ \pm}(\sigma)=S_{K^{*}}^{ \pm}(\beta(\sigma)) \text { and } S_{K^{*}}^{ \pm}\left(p_{\tau, \sigma}\right)=f_{\tau, \sigma},
$$

where $f_{\tau, \sigma}: S_{K^{*}}^{ \pm}(\tau) \rightarrow S_{K^{*}}^{ \pm}(\sigma)$ is induced by mapping those $\beta_{j}$ to 1 for which $v_{j} \in \tau \backslash \sigma$. 
In this case the Laurent face algebra $F_{K}[\Sigma]=F_{K}[\beta ; \Sigma]$ is the limit of $S_{K^{*}}^{ \pm}$, and is additively generated by those monomials $(1-\beta)^{J}:=\left(1-\beta_{1}\right)^{j_{1}} \cdots\left(1-\beta_{m}\right)^{j_{m}}$ whose support $\prod_{j_{k} \neq 0}\left(1-\beta_{k}\right)$ is $(1-\beta)_{\sigma}$ for some cone $\sigma$, where $J=\left(j_{1}, \ldots, j_{m}\right)$ lies in $\mathbb{Z}^{m}$. There is therefore a canonical isomorphism

$$
S_{K^{*}}^{ \pm}(\beta) /\left((1-\beta)_{\omega}: \omega \notin \Sigma\right) \stackrel{\cong}{\longrightarrow} F_{K}[\beta ; \Sigma]
$$

of graded $S_{K^{*}}^{ \pm}(\beta)$-algebras.

A similar construction is possible for the $M U_{T^{n}}$-analogue $F_{M U}[\Sigma]$, and for the versions involving formal power series used below. In the case of cohomology, $F_{H R}[\Sigma]$ coincides with the standard face ring $R[\Sigma]$, so we retain the latter notation.

We show that $P_{K}(\alpha ; \Sigma)$ is isomorphic to $F_{K}[\beta ; \Sigma]$ by appealing to the defining diagrams; in the context of algebraic $K$-theory, a proof has long been available [VV].

By analogy with (5.3), we consider the $n \times m$ matrix

$$
\xi=\xi_{\Sigma}:=\left(\begin{array}{lll}
v_{1} & \cdots & v_{m}
\end{array}\right) .
$$

This notation is consistent with Remark 4.22, for we may view $\xi$ as a map $\Sigma^{\prime} \rightarrow \Sigma$ of fans; the rays of $\Sigma^{\prime}$ are the standard basis vectors in $\mathbb{R}^{m}$, and its cones $\sigma^{\prime}:=\left\{e_{i_{1}}, \ldots, e_{i_{k}}\right\}$ correspond bijectively to the cones $\sigma:=\left\{v_{i_{1}}, \ldots, v_{i_{k}}\right\}$ of $\Sigma$. For any $n$-dimensional $\sigma$ we write $\xi_{\sigma}$ for the $n \times n$ submatrix of $\xi$ whose columns generate $\sigma$. The smoothness of $\Sigma$ guarantees that every $\xi_{\sigma}$ is invertible over $\mathbb{Z}$. So $\xi$ defines an epimorphism $\mathbb{Z}^{m} \rightarrow \mathbb{Z}^{n}$, and $\xi^{t r}$ induces monomorphisms $S_{R}(x) \rightarrow S_{R}(y)$ and $S_{K^{*}}^{ \pm}(\alpha) \rightarrow S_{K^{*}}^{ \pm}(\beta)$ of graded rings; the latter maps $\alpha^{J}$ to $\beta^{\xi^{t r} J}$ for any $J \in \mathbb{Z}^{n}$.

Proposition 7.8. For any smooth polytopal fan $\Sigma$, the matrix $\xi$ induces a natural isomorphism

$$
\xi^{*}: P_{K}(\alpha ; \Sigma) \longrightarrow F_{K}[\beta ; \Sigma]
$$

of algebras over $\xi^{t r}: S_{K^{*}}^{ \pm}(\alpha) \rightarrow S_{K^{*}}^{ \pm}(\beta)$.

Proof. The epimorphism $\xi: \mathbb{Z}^{m} \rightarrow \mathbb{Z}^{n}$ maps the generator $e_{j}$ to the ray $v_{j}$, for all $1 \leq j \leq m$. It therefore induces an isomorphism $\xi^{*}(\sigma): K V(\sigma) \rightarrow S_{K^{*}}^{ \pm}(\sigma)$ of algebras over $\xi^{t r}$, defined by $\xi^{*}(\sigma)\left(\alpha^{J}\right)=\beta^{\xi(\sigma)^{t r} J}$ for any $J \in \mathbb{Z}^{n}$. Moreover, $f_{\tau, \sigma} \cdot \xi^{*}(\tau)=\xi^{*}(\sigma) \cdot q_{\tau, \sigma}$ for every morphism $\tau \supseteq \sigma$ in $\operatorname{CAT}(\Sigma)$, because $\xi_{\tau} \supseteq \xi_{\sigma}$ as submatrices of $\xi$.

So $\xi^{*}$ is a natural isomorphism of diagrams, and induces the required isomorphism of limits.

Corollary 7.9. For any smooth polytopal fan $\Sigma$, there is an isomorphism $K_{T^{n}}^{*}\left(X_{\Sigma}\right) \rightarrow F_{K}[\beta ; \Sigma]$ of algebras over $\xi^{t r}$.

Proof. Combine Corollary 7.2 with Proposition 7.8.

Example 7.10. If $\xi$ is the $n \times(n+1)$ matrix $\left(-1 I_{n}\right)$, then $X_{\Sigma}$ is $\mathbb{C} P^{n}$ and there is an isomorphism

$$
K_{T^{n}}^{*}\left(\mathbb{C} P^{n}\right) \longrightarrow S_{K^{*}}^{ \pm}\left(\beta_{0}, \ldots, \beta_{n}\right) /\left(\prod_{j=0}^{n}\left(1-\beta_{j}\right)\right)
$$

of algebras over $\xi^{t r}$. An equivalent formula appears, for example, in [GW].

Finally, for any smooth polytopal fan $\Sigma$, we describe the Borel equivariant $K$-theory of $X_{\Sigma}$ in terms of the face ring $F_{K}[[\delta ; \Sigma]]$, whose indeterminates are 0 -dimensional. This is an algebra over $K^{*}[[\delta]]$, and is the limit of the $\Sigma^{o p}$-diagram that assigns $K^{*}\left[\left[\delta_{1}, \ldots, \delta_{m}\right]\right] /\left(\delta_{\omega}: \omega \notin \sigma\right)$ to each cone $\sigma \in \Sigma$. It is also the completion of $F_{K}[\beta ; \Sigma]$ at the augmentation ideal $\left(1-\beta_{1}, \ldots, 1-\beta_{m}\right)$, where $\delta_{j}=1-\beta_{j}$ for all $1 \leq j \leq m$.

We retain the notation of (7.7), observing that $\xi^{t r}$ of Proposition 7.8 extends to a homomorphism $\xi^{t r}: K^{*}[[\gamma]] \rightarrow K^{*}[[\delta]]$. 
Proposition 7.11. For any smooth polytopal fan $\Sigma$, there are isomorphisms

$$
K^{*}\left(E T^{n} \times_{T^{n}} X_{\Sigma}\right) \longrightarrow P_{K_{B}}(\gamma ; \Sigma) \stackrel{\xi^{*}}{\longrightarrow} F_{K}[[\delta ; \Sigma]]
$$

of algebras over $K^{*}[[\gamma]]$ and $\xi^{t r}$ respectively.

Proof. The first isomorphism is the completion of Corollary 7.2.2, and the second is the completion of Proposition 7.8 .

\section{REFERENCES}

[A] J. Frank Adams, Stable homotopy and generalised homology. Chicago Lectures in Mathematics, University of Chicago Press, Chicago, Ill. - London, 1974.

[ALR] Alejandro Adem, Johann Leida and Yongbin Ruan, Orbifolds and Stringy Topology. Cambridge Tracts in Mathematics 171. Cambridge University Press, Cambridge, 2007.

[AlPu] Christopher Allday and Volke Puppe, Cohomological methods in transformation groups. Cambridge Studies in Advanced Mathematics, 32. Cambridge University Press, Cambridge, 1993.

[AnPa] David Anderson and Sam Payne, Operational K-theory. arXiv:1301.0425.

[AM] Michael F. Atiyah and Ian G. MacDonald, Introduction to commutative algebra. Addison-Wesley Publishing Co., Reading Mass. - London - Don Mills, Ont., 1969.

[AS1] Michael F. Atiyah and Graeme Segal, Equivariant K-theory and completion. J. Differential Geometry 3 (1969), 1-18.

[AS2] Michael F. Atiyah and Graeme Segal, Twisted K-theory. Ukr. Mat. Visn. 1 (2004), no. 3, 287-330; translation in Ukr. Math. Bull. 1 (2004), no. 3, 291-334.

[BFNR] Anthony Bahri, Matthias Franz, Dietrich Notbohm and Nigel Ray, The classification of weighted projective spaces. Fund. Math. 220 (2013), no. 3, 217-226.

[BFR1] Anthony Bahri, Matthias Franz and Nigel Ray, The equivariant cohomology ring of weighted projective space. Math. Proc. Cambridge Philos. Soc. 146 (2009), no. 2, 395-405.

[BFR2] Anthony Bahri, Matthias Franz and Nigel Ray, Weighted projective spaces and iterated Thom spaces. Osaka J. Math. 51 (2014), no. 1, 89-119.

[B] Michel Brion, The structure of the polytope algebra. Tohoku Math. J. (2) 49 (1997), no. 1, 1-32.

[CdS $\quad$ A. Cannas da Silva, Lectures on symplectic geometry (Corrected 2nd printing). Lecture Notes in Mathematics 1764, Springer-Verlag, Berlin, 2008.

[CGK] Michael Cole, John P.C. Greenlees and Igor Kriz, The universality of equivariant complex bordism. Math. Z. 239 (2002), no. 3, 455-475.

[C] Gustavo Comezaña, Calculations in complex equivariant bordism. Chapter XXVIII of Equivariant Homotopy and Cohomology Theory. CBMS Regional Conference Series in Mathematics Vol. 91, 333-352, AMS Publications, Providence, RI, 1996.

[CM] Gustavo Comezaña and J. Peter May, A completion theorem in complex cobordism. Chapter XXVII of Equivariant Homotopy and Cohomology Theory. CBMS Regional Conference Series in Mathematics Vol. 91, 327-332, AMS Publications, Providence, RI, 1996.

[CLS] David A. Cox, John B. Little and Henry K. Schenck, Toric Varieties. Graduate Studies in Mathematics Volume 124. American Mathematical Society, Providence, RI, 2011.

[De] Thomas Delzant, Hamiltoniens périodiques et images convexes de l'application moment. Bull. Soc. Math. France 116 (1988), no. 3, 315-339.

[tD] Tammo tom Dieck, Bordism of $G$-manifolds and integrality theorems. Topology 9 (1970), 345-358.

[Do] Igor Dolgachev, Weighted projective varieties. Group actions and vector fields (Vancouver, B.C., 1981), 34-71, Lecture Notes in Math., 956, Springer, Berlin, 1982.

[Du] Delphine Dupont, Faisceaux pervers sur les variétés toriques lisses. arXiv:1003.3199v1.

[Fr] Matthias Franz, Describing toric varieties and their equivariant cohomology. Colloq. Math. 121 (2010), no. $1,1-16$.

[Fu] William Fulton, Introduction to Toric Varieties. Annals of Mathematics Studies 131, Princeton University Press, Princeton, NJ, 1993.

[GoKa] José Luis González and Kalle Karu, Bivariant algebraic cobordism. arXiv:11301.4210v1.

[GKM] Mark Goresky, Robert Kottwitz and Robert MacPherson, Equivariant cohomology, Koszul duality, and the localization theorem. Invent. Math. 131 (1998), no. 1, 25-83.

[GW] John P. C. Greenlees and Gareth R. Williams, Poincaré duality for $K$-theory of equivariant complex projective spaces. Glasg. Math. J. 50 (2008), no. 1, 111-127.

[HaHeHo] Megumi Harada, Andrés Henriques and Tara Holm, Computation of generalized equivariant cohomologies of Kac-Moody flag varieties. Adv. Math. 197 (2005), no. 1, 198-221. 
[HaL] Megumi Harada and Gregory Landweber, The $K$-theory of abelian symplectic quotients. Math. Res. Lett. 15 (2008), no. 1, 57-72.

[Haz] Michiel Hazewinkel, Formal Groups and Applications. AMS Chelsea, 2012.

[Hi] Peter Hilton, General cohomology theory and K-theory. London Mathematical Society Lecture Note Series 1, Cambridge University Press, London - New York, 1971.

[HoM] Tara Holm and Tomoo Matsumura, Equivariant cohomology for Hamiltonian torus actions on symplectic orbifolds. Transform. Groups 17 (2012), no. 3, 717-746.

[Hu] Dale Husemoller, Fiber Bundles (third edition). Graduate Texts in Mathematics 20, Springer-Verlag, 1994.

[Kan] Tamafumi Kaneyama, Torus-equivariant vector bundles on projective spaces. Nagoya Math. J. 111 (1988), 25-40.

[Kaw] Tetsuro Kawasaki, Cohomology of twisted projective spaces and lens complexes. Math. Ann. 206 (1973), 243-248.

[Kl] Alexander A. Klyachko, Equivariant bundles over toric varieties. (Russian) Izv. Akad. Nauk SSSR Ser. Mat. 53 (1989), no. 5, 1001-1039, 1135; translation in Math. USSR-Izv. 35 (1990), no. 2, 337-375.

[KK] Valentina Kiritchenko and Amalendu Krishna, Equivariant cobordism of flag varieties and of symmetric varieties arXiv:math/1104.1089.

[KU] Amalendu Krishna and Vikraman Uma, The algebraic cobordism ring of toric varieties. Int. Math. Res. Not. IMRN (2013), no. 23, 5426-5464.

[L] Eugene Lerman, Orbifolds as stacks? arXiv:0806.4160v2.

[LY] Chen-Hao Liu and Shing-Tung Yau, On the splitting type of an equivariant vector bundle over a toric manifold. arXiv:math/0002031.

[Ma] J. Peter May (editor), Equivariant Homotopy and Cohomology Theory. CBMS Regional Conference Series in Mathematics 91, 333-352. Published for the Conference Board of the Mathematical Sciences, Washington, DC; by the American Mathematical Society, Providence, RI, 1996.

[Moe] Ieke Moerdijk, Orbifolds as groupoids: an introduction. In Orbifolds in Mathematics and Physics (Madison WI, 2001). Contemp. Math., 310, Amer. Math. Soc., Providence, RI, 2002.

[Mor] Robert Morelli, The K-theory of a toric variety. Adv. Math. 100 (1993), no. 2, 154-182.

[NY] Yasuzo Nishimura and Zen-ichi Yosimura, The quasi $K O_{*}$-types of weighted projective spaces. J. Math. Kyoto Univ. 37 (1997), no. 2, 251-259.

[Od] Tadao Oda, Convex bodies and algebraic geometry. An introduction to the theory of toric varieties. Translated from the Japanese. Ergebnisse der Mathematik und ihrer Grenzgebieter (3) [Results in Mathematics and Related Areas (3)], 15. Springer-Verlag, Berlin, 1988.

[Ok] Christian Okonek, Der Conner-Floyd-Isomorphismus für Abelsche Gruppen. Math. Z. 179 (1982), no. 2, 201-212.

[ON] Beverley O'Neil, Cellular structures and stunted weighted projective space. PhD Thesis, University of Manchester, 2013.

[PRV] Taras Panov, Nigel Ray and Rainer Vogt, Colimits, Stanley-Reisner algebras, and loop spaces. (English Summary) Categorical decoposition techniques in algebraic topology (Isle of Skye, 2001), 261-291, Progr. Math., 215, Birkhäuser, 2004.

[P] Sam Payne, Toric vector bundles, branched covers of fans, and the resolution property. J. Algebraic Geom. 18 (2009), no. 1, 1-36.

[RKR] Ioanid Rosu (with an appendix by Allen Knutson and Ioanid Rosu), Equivariant $K$-theory and equivariant cohomology. Math. Z. 243 (2003), no. 3, 423-448.

[Se] Graeme Segal, Equivariant K-theory. Inst. Hautes Études Sci. Publ. Math. 34 (1968), 129-151.

[Si] Dev Sinha, Computations of complex equivariant bordism rings. Amer. J. Math. 123 (2001), no. 4, 577-605.

[VV] Gabriele Vezzosi and Angelo Vistoli, Higher algebraic $K$-theory for actions of diagonalizable groups. Invent. Math. 153 (2003), no. 1, 1-44.

[WZZ] Volkmar Welker, Günter M. Ziegler and Rade T. Živaljević, Homotopy colimits - comparison lemmas for combinatorial applications. J. Reine Angew. Math. 509 (1999), 117-149. 
Department of Mathematics and Statistics, McMaster University, 1280 Main Street West, Hamilton, Ontario L8S4K1, Canada

E-mail address: Megumi.Harada@math.mcmaster.ca

Department of Mathematics, Malott Hall, Cornell University, Ithaca, New York 14853-4201, USA

E-mail address: tsh@math.cornell.edu

School of Mathematics, University of Manchester, Oxford Road, Manchester M13 9PL, UK

E-mail address: nigel.ray@manchester.ac.uk

Department of Mathematics and Statistics, The Open University, Walton Hall, Milton Keynes MK7 6AA, UK

E-mail address: G.R.Williams@Open.ac.uk 